\title{
Hedging under worst-case-scenario in a market driven by time-changed Lévy noises
}

\author{
Giulia Di Nunno* $\quad$ Erik Hove Karlsen ${ }^{\dagger}$
}

Version: April 20, 2015

\begin{abstract}
In an incomplete market driven by time-changed Lévy noises we consider the problem of hedging a financial position coupled with the underlying risk of model uncertainty. Then we study hedging under worst-case-scenario. The proposed strategies are not necessarily self-financing and include the interplay of a cost process to achieve the perfect hedge at the end of the time horizon. The hedging problem is tackled in the framework of stochastic differential games and it is treated via backward stochastic differential equations. Two different information flows are considered and the solutions compared.
\end{abstract}

Keywords: model uncertainty, hedging, BSDEs, stochastic differential games, time-change, martingale random fields.

MSC2010 Classification: 93E20, 91G80, 60G60, 60G48

JEL Classification: G11, C70

\section{Introduction}

In a stylized incomplete market on the time horizon $[0, T](T>0)$, we consider the problem of hedging a contingent claim coupled with the underlying risk of an uncertain model description. This is referred to as model ambiguity in the literature, see e.g. [CE02. We choose to consider a conservative evaluation of such exposure to model ambiguity by measuring the robustness of the strategy in terms of a worst-case-scenario risk measure. In fact, on the complete filtered probability space $(\Omega, \mathcal{F}, \mathbb{P}), \mathbb{M}=\left\{\mathcal{M}_{t}, t \in[0, T]\right\}$, we fix the dynamic coherent risk measure

$$
\rho_{t}(\xi):=\underset{\mathbb{Q} \in \mathcal{Q}_{\mathbb{M}}}{\operatorname{ess} \sup _{\mathbb{Q}}} \mathbb{E}_{\mathbb{Q}}\left[-\xi \mid \mathcal{M}_{t}\right], \quad t \in[0, T],
$$

where $\mathcal{Q}_{\mathbb{M}}$ is the set of all scenarios considered, which are given by the probability measures $\mathbb{Q}$ equivalent to $\mathbb{P}$ on the future outcomes $\left(\Omega, \mathcal{M}_{T}\right)$. The risk measure $\rho$ is well-defined and finite for all financial positions $\xi$ integrable with respect to all $\mathbb{Q} \in \mathcal{Q}_{\mathbb{M}}$. This risk measure is naturally connected to the concept of worst-case-scenario, associating the risk evaluation with the highest expected value on the downside of the position over all $\mathbb{Q} \in \mathcal{Q}_{\mathbb{M}}$. For this reason it is also a conservative evaluation of the risk exposure.

\footnotetext{
${ }^{*}$ CMA and Department of Mathematics, University of Oslo, P.O. Box 1053 Blindern, N-0316 Oslo, and Department of Business and Management Science, NHH, Helleveien 30, N-5045 Bergen. Email: giulian@math.uio.no

${ }^{\dagger}$ Department of Mathematics, University of Oslo, P.O. Box 1053 Blindern, N-0316 Oslo. Email: erikhk@math.uio.no.
} 
The financial market presents two investment possibilities: a saving account with price dynamics given by

$$
d S_{t}^{(0)}=r_{t} S_{t}^{(0)} d t, \quad S_{0}^{(0)}=1,
$$

which is used as numéraire, and a stock with price dynamics of the type

$$
d S_{t}^{(1)}=\alpha_{t} S_{t}^{(1)} d t+\sigma_{t} S_{t}^{(1)} d B_{t}+\int_{\mathbb{R}_{0}} \gamma_{t}(z) S_{t^{-}}^{(1)} \tilde{H}(d t, d z), \quad S_{0}^{(1)}>1 .
$$

The driving noises $B$ and $\tilde{H}$ are a doubly stochastic Gaussian and Poisson type measures, respectively related to a time-changed Brownian and Poisson process. The parameters $r, \alpha$, $\sigma, \gamma$ are càglàd adapted stochastic processes and fields. To ensure the existence of a square integrable positive solution and to allow further analysis, we assume that $\left|r_{t}\right|<C$ (for some $C>0) \mathbb{P} \times d t$-a.e., $\gamma_{t}(z)>-1 \mathbb{P} \times \Lambda$-a.e., and

$$
\mathbb{E}\left[\int_{0}^{T}\left\{\left|\alpha_{t}\right|+\sigma_{t}^{2} \lambda_{t}^{B}+\int_{\mathbb{R}_{0}}\left|\ln \left(1+\gamma_{t}(z)\right)-\gamma_{t}(z)\right| \nu(d z) \lambda_{t}^{H}\right\} d t\right]<\infty .
$$

The elements $\nu,\left(\lambda^{B}, \lambda^{H}\right)$, and $\Lambda$ are associated to the behaviour of the jumps in these dynamics and the intensity of the (stochastic) time distortion applied, see the next section for details.

Price dynamics of this type include various well-known stochastic volatility price models such as CGMY03, where time-change of a pure jump Lévy process is used to take the erratic behaviour of volatility into account by mimicking the transition between a real-time clock to a transactions-time clock. See also e.g. [BNNS02, Hes93, HW87, SS91] where the stochastic volatility models lead to dynamics driven by time-changed Brownian motions, which are the doubly stochastic Gaussian noises in this paper. Also in the credit risk literature we can find examples of price dynamics of the type above. See e.g. [Lan98, where doubly stochastic Poisson processes, also called the Cox processes, are largely used in the modelling of prices subject to default risk.

In the market above, we consider a financial claim $F \in L^{2}\left(\Omega, \mathcal{M}_{T}, \mathbb{P}\right)$ with payoff at $T>0$ and the M-predictable hedging strategies represented by the triple $\left(\pi, V^{\pi}, C^{\pi}\right)$, i.e. $\pi_{t}$ is the wealth invested in the stock at $t$ representing the market portfolio, $V_{t}^{\pi}$ the value of the strategy on the market, and $C_{t}^{\pi}$ is the cost process. We denote $V_{0}^{\pi}=v>0$. We assume that

$$
\begin{aligned}
d V_{t}^{\pi} & =\frac{\pi_{t}}{S_{t^{-}}^{(1)}} d S_{t}^{(1)}+\frac{V_{t}^{\pi}-\pi_{t}}{S_{t}^{(0)}} d S_{t}^{(0)} \\
& \left.=\left(V_{t}^{\pi} r_{t}+\pi_{t}\left(\alpha_{t}-r_{t}\right)\right) d t+\pi_{t} \sigma_{t} d B_{t}+\int_{\mathbb{R}_{0}} \pi_{t} \gamma_{t}(z) \tilde{H}(d t, d z)\right)
\end{aligned}
$$

and that $C_{t}^{\pi}=\rho_{t}\left(e^{-\int_{t}^{T} r_{s} d s}\left(V_{T}^{\pi}-F\right)\right)$. We assume that (1.4) admits a unique strong solution for any admissible $\pi$ (see e.g. JJac79] for conditions). Moreover, we require that the solution is square integrable, and for this we assume

$$
\mathbb{E}\left[\int_{0}^{T}\left\{\left|\alpha_{t}-r_{t}\right|\left|\pi_{t}\right|+\left|\alpha_{t} \sigma_{t}\right|^{2} \lambda_{t}^{B}+\int_{\mathbb{R}_{0}}\left|\pi_{t} \gamma_{t}(z)\right|^{2} \nu(d z) \lambda_{t}^{H}\right\} d t\right]<\infty .
$$

Moreover, the process $C^{\pi}$ is also assumed square integrable.

The process $Y_{t}^{\pi}:=V_{t}^{\pi}+C_{t}^{\pi}, t \geq 0$, is called the (total) price of the strategy. The hedging of $F$ is obtained for a strategy $\left(\hat{\pi}, V^{\hat{\pi}}, C^{\hat{\pi}}\right)$ that yields $Y_{T}^{\hat{\pi}}=F$. Note that $Y_{0}^{\pi}=v+C_{0}^{\pi}$ is $\mathcal{M}_{0^{-}}$ measurable and, if the hedging strategy has $C^{\pi} \equiv 0$, then the market investments are enough 
to self-finance the hedge. It is only in a complete market that it is possible to hedge all claims with these self-financing strategies.

We observe that for any hedging strategy $\left(\pi, V^{\pi}, C^{\pi}\right)$ with $Y_{T}^{\pi}=F$ we have

$$
\rho_{t}\left(e^{-\int_{t}^{T} r_{s} d s}\left(Y_{T}^{\pi}-F\right)\right)=0, \quad t \in[0, T] .
$$

This means that the risk given by the spread between the discounted final strategy price and the actual claim is zero according to the risk measure given, and actually all coherent risk measures.

Hereafter, we consider the problem of finding a hedging strategy $\left(\hat{\pi}, V^{\hat{\pi}}, C^{\hat{\pi}}\right)$ for $F$ such that $Y_{t}^{\hat{\pi}}=Y_{t}, t \in[0, T]$, where

$$
Y_{t}:=\underset{\pi \in \Pi_{\mathbb{M}}}{\operatorname{essinf}} \rho_{t}\left(e^{-\int_{t}^{T} r_{s} d s}\left(V_{T}^{\pi}-F\right)-V_{t}^{\pi}\right), \quad t \in[0, T] .
$$

Hence the strategy $\hat{\pi}$ minimizes the risk associated to the total price. Clearly $Y_{T}^{\hat{\pi}}=Y_{T}=F$. Here above, the set $\Pi_{\mathbb{M}}$ denotes the admissible strategies.

Considering the risk measure (1.1), we can write the problem (1.6) in the following way:

$$
\begin{aligned}
Y_{t}^{\hat{\pi}}=Y_{t}= & \underset{\pi \in \Pi_{\mathbb{M}}}{\operatorname{essinf}} \underset{\mathbb{Q} \in \mathcal{Q}_{\mathbb{M}}}{\operatorname{ess} \sup } E_{\mathbb{Q}}\left[-\left(e^{-\int_{t}^{T} r_{s} d s} V_{T}^{\pi}-V_{t}^{\pi}-e^{-\int_{t}^{T} r_{s} d s} F\right) \mid \mathcal{M}_{t}\right] \\
= & \underset{\pi \in \Pi_{\mathbb{M}}}{\operatorname{essinf}} \underset{\mathbb{Q} \in \mathcal{Q}_{\mathbb{M}}}{\operatorname{ess} \sup _{\mathbb{Q}}} E_{\mathbb{Q}}\left[e^{-\int_{t}^{T} r_{s} d s} F-\int_{t}^{T} e^{-\int_{t}^{s} r_{u} d u} \pi_{s}\left(\alpha_{s}-r_{s}\right) d s\right. \\
& -\int_{t}^{T} e^{-\int_{t}^{s} r_{u} d u} \pi_{s} \sigma_{s} d B_{s} \\
& \left.-\int_{t}^{T} \int_{\mathbb{R}_{0}} e^{-\int_{t}^{s} r_{u} d u} \pi_{s} \gamma_{s}(z) \tilde{H}(d s, d z) \mid \mathcal{M}_{t}\right]
\end{aligned}
$$

A solution to the problem (1.7) corresponds to finding $(\hat{\pi}, \hat{\mathbb{Q}}) \in \Pi_{\mathbb{M}} \times \mathcal{Q}_{\mathbb{M}}$ such that

$$
\begin{aligned}
Y_{t}=E_{\hat{\mathbb{Q}}}[ & e^{-\int_{t}^{T} r_{s} d s} F-\int_{t}^{T} e^{-\int_{t}^{s} r_{u} d u} \hat{\pi}_{s}\left(\alpha_{s}-r_{s}\right) d s \\
& -\int_{t}^{T} e^{-\int_{t}^{s} r_{u} d u} \hat{\pi}_{s} \sigma_{s} d B_{s} \\
& \left.\quad-\int_{t}^{T} \int_{\mathbb{R}_{0}} e^{-\int_{t}^{s} r_{u} d u} \hat{\pi}_{s} \gamma_{s}(z) \tilde{H}(d s, d z) \mid \mathcal{M}_{t}\right], \quad t \in[0, T] .
\end{aligned}
$$

The cost of the hedge is then $C_{t}^{\hat{\pi}}=E_{\hat{\mathbb{Q}}}\left[e^{-\int_{t}^{T} r_{s} d s}\left(F-V_{T}^{\hat{\pi}}\right) \mid \mathcal{M}_{t}\right]$. To have a unique description of the optimal strategy, we set $E_{\hat{\mathbb{Q}}}\left[C_{0}^{\hat{\pi}}\right]=0$ and $v=E_{\hat{\mathbb{Q}}}\left[Y_{0}^{\hat{\pi}}\right]$.

This kind of approach to hedging is treated in Del12 (see also Del13) in the context of a financial market driven by a Brownian motion and an insurance payment process driven by a Poisson process. See also BP15 for a study of a similar problem within an insurance perspective, but Brownian driven dynamics. We also refer to Kar14, where a first study of this problem is given in the context of a market driven by a Brownian motion and a doubly stochastic Poisson noise. Comparatively, in the present paper we consider a more general market model and a substantially different structure of admissible strategies. 
The admissible scenarios are described by a measure change via shift transformation. With respect to this, we suggest a version of the Girsanov theorem that explicitely deals with timechange. We note that, in this context, the measure change is not structure preserving in general. The hedging problem (1.7)-(1.8) is tackled using backward stochastic differential equations (BSDEs) and stochastic differential games. Our study is carried through in the context of two different filtrations: $\mathbb{M}=\mathbb{F}$, which is substantially the information flow generated by the noises, and $\mathbb{M}=\mathbb{G}$, which is the filtration that, additionally to $\mathbb{F}$, includes initial knowledge of the time-change process. These two settings lead to different BSDEs depending on their measurability properties. We treat the solutions exploiting the martingale random field properties of the driving noises and, in the case of $\mathbb{G}$, we also rely on the better explicit structure of the noise (which allows for a more explicit stochastic representation theorem).

In the case of information flow $\mathbb{G}$, BSDEs driven by doubly stochastic Lévy noises are treated in DS14. We also mention that these integral representation theorems are studied in [DS13] with different approaches: via chaos expansions and via the non-anticipating derivative (see also [DR07 for a review on stochastic derivation). As for filtration $\mathbb{F}$, we rely on the general results of [CFS08], which we adapt to the random field set-up.

Even though we can regard the information flow $\mathbb{F}$ as partial with respect to $\mathbb{G}$, the problems presented here are not the same as in the study on BSDEs with partial information, see e.g. CCR14 in the case of mean-variance hedging.

Finally, we remark that the hedging criteria we consider differs from mean-variance hedging in the objective function to minimize: mean-variance hedging identifies the strategy by minimizing the quadratic cost, see e.g. [JMSS12] in the context of prices modeled by general semimartingales, and Lim05] for the case of dynamics driven by a Brownian motion and doubly stochastic Poisson noises.

The paper is structured as follows: the next section provides details about the framework and the BSDEs considered. In Section 3, we study shift transformations, while Section 4 is dedicated to the actual solution of the hedging problem in the two information flows considered. Section 5 concludes with comments on the results obtained.

\section{The framework and preliminary results}

Hereafter, we give full detail of the noises considered in (1.3) and the stochastic structures used. For this we refer to DS14] and DS13]. In particular, we apply stochastic integration with respect to martingale random fields, see [DE10] and CW75].

\subsection{The random measures and their properties}

Let $(\Omega, \mathcal{F}, \mathbb{P})$ be a complete probability space and $\mathbb{X}:=[0, T] \times \mathbb{R}$. We will consider $\mathbb{X}=$ $([0, T] \cup\{0\}) \cup\left([0, T] \times \mathbb{R}_{0}\right)$, where $\mathbb{R}_{0}:=\mathbb{R} \backslash\{0\}$ and $T>0$. Clearly, $[0, T] \cup\{0\} \simeq[0, T]$. Denote $\mathcal{B}_{\mathbb{X}}$ the Borel $\sigma$-algebra on $\mathbb{X}$. Whenever we write $\Delta \subset \mathbb{X}$, we intend a set $\Delta$ in $\mathcal{B}_{\mathbb{X}}$.

The two dimensional stochastic process $\lambda:=\left(\lambda^{B}, \lambda^{H}\right)$ represents the intensity of the stochastic time distortion applied in the noise. Each component $\lambda^{l}$ for $l=B, H$, satisfies

i) $\lambda_{t}^{l} \geq 0 \mathbb{P}$-a.s. for all $t \in[0, T]$,

ii) $\lim _{h \rightarrow 0} \mathbb{P}\left(\left|\lambda_{t+h}^{l}-\lambda_{t}^{l}\right| \geq \epsilon\right)=0$ for all $\epsilon>0$ and almost all $t \in[0, T]$,

iii) $\mathbb{E}\left[\int_{0}^{T} \lambda_{t}^{l} d t\right]<\infty$. 
Denote the space of all processes $\lambda:=\left(\lambda^{B}, \lambda^{H}\right)$ satisfying i), ii) and iii) by $\mathcal{L}$. Correspondingly, we define the random measure $\Lambda$ on $\mathbb{X}$ by

$$
\Lambda(\Delta):=\int_{0}^{T} \mathbf{1}_{\Delta}(t, 0) \lambda_{t}^{B} d t+\int_{0}^{T} \int_{\mathbb{R}_{0}} \mathbf{1}_{\Delta}(t, z) \nu(d z) \lambda_{t}^{H} d t, \quad \Delta \in \mathbb{X} .
$$

Here $\nu$ is a (deterministic) $\sigma$-finite measure on the Borel sets of $\mathbb{R}_{0}$ satisfying

$$
\int_{\mathbb{R}_{0}} z^{2} \nu(d z)<\infty
$$

We denote the $\sigma$-algebra generated by the values of $\Lambda$ by $\mathcal{F}^{\Lambda}$. Furthermore, $\Lambda^{H}$ denotes the restriction of $\Lambda$ to $[0, T] \times \mathbb{R}_{0}$ and $\Lambda^{B}$ the restriction of $\Lambda$ to $[0, T] \times\{0\}$. Hence $\Lambda(\Delta)=$ $\Lambda^{B}(\Delta \cap[0, T] \times\{0\})+\Lambda^{H}\left(\Delta \cap[0, T] \times \mathbb{R}_{0}\right), \Delta \subseteq \mathbb{X}$. Here below we introduce the noises driving the stochastic dynamics.

Definition 2.1. $B$ is a signed random measure on the Borel sets of $[0, T] \times\{0\}$, satisfying

A1) $\mathbb{P}\left(B(\Delta) \leq x \mid \mathcal{F}^{\Lambda}\right)=\mathbb{P}\left(B(\Delta) \leq x \mid \Lambda^{B}(\Delta)\right)=\Phi\left(\frac{x}{\sqrt{\Lambda^{B}(\Delta)}}\right), x \in \mathbb{R}, \Delta \subseteq[0, T] \times\{0\}$,

A2) $B\left(\Delta_{1}\right)$ and $B\left(\Delta_{2}\right)$ are conditionally independent given $\mathcal{F}^{\Lambda}$ whenever $\Delta_{1}$ and $\Delta_{2}$ are disjoint sets.

Here $\Phi$ stands for the cumulative probability distribution function of a standard normal random variable.

$H$ is a random measure on the Borel sets of $[0, T] \times \mathbb{R}_{0}$, satisfying

A3) $\mathbb{P}\left(H(\Delta)=k \mid \mathcal{F}^{\Lambda}\right)=\mathbb{P}\left(H(\Delta)=k \mid \Lambda^{H}(\Delta)\right)=\frac{\Lambda^{H}(\Delta)^{k}}{k !} e^{-\Lambda^{H}(\Delta)}, k \in \mathbb{N}, \Delta \subseteq[0, T] \times \mathbb{R}_{0}$,

A4) $H\left(\Delta_{1}\right)$ and $H\left(\Delta_{2}\right)$ are conditionally independent given $\mathcal{F}^{\Lambda}$ whenever $\Delta_{1}$ and $\Delta_{2}$ are disjoint sets.

Furthermore, we assume that

A5) $B$ and $H$ are conditionally independent given $\mathcal{F}^{\Lambda}$.

Substantially, conditional on $\Lambda$, we have that $B$ is a Gaussian random measure and $H$ is a Poisson random measure. We refer to [Gri75] or [Kal97] for the existence of the above conditional distributions.

Let $\tilde{H}:=H-\Lambda^{H}$ be the signed random measure given by

$$
\tilde{H}(\Delta)=H(\Delta)-\Lambda^{H}(\Delta), \quad \Delta \subseteq[0, T] \times \mathbb{R}_{0} .
$$

Definition 2.2. We define the signed random measure $\mu$ on the Borel subsets of $\mathbb{X}$ by

$$
\mu(\Delta):=B(\Delta \cap[0, T] \times\{0\})+\tilde{H}\left(\Delta \cap[0, T] \times \mathbb{R}_{0}\right), \quad \Delta \subseteq \mathbb{X} .
$$

The random measures $B$ and $H$ are related to a specific form of time-change for Brownian motion and pure jump Lévy process. More specifically define $B_{t}:=B([0, t] \times\{0\}), \Lambda_{t}^{B}:=\int_{0}^{t} \lambda_{s}^{B} d s$, $\eta_{t}:=\int_{0}^{t} \int_{\mathbb{R}_{0}} z \tilde{H}(d s, d z)$ and $\hat{\Lambda}_{t}^{H}:=\int_{0}^{t} \lambda_{s}^{H} d s$, for $t \in[0, T]$. 
We can immediately see the role that the time-change processes $\Lambda^{B}$ and $\hat{\Lambda}^{H}$ play by studying the characteristic function of $B$ and $\eta$. In fact, from A1) and A3) we see that the conditional characteristic functions of $B_{t}$ and $\eta_{t}$ are given by

$$
\begin{aligned}
\mathbb{E}\left[e^{i c B_{t}} \mid \mathcal{F}^{\Lambda}\right] & =\exp \left\{\int_{0}^{t} \frac{1}{2} c^{2} \lambda_{s}^{B} d s\right\}=\exp \left\{\frac{1}{2} c^{2} \Lambda_{t}^{B}\right\}, \quad c \in \mathbb{R}, \\
\mathbb{E}\left[e^{i c \eta_{t}} \mid \mathcal{F}^{\Lambda}\right] & =\exp \left\{\int_{0}^{t} \int_{\mathbb{R}_{0}}\left[e^{i c z}-1-i c z\right] \nu(d z) \lambda_{s}^{H} d s\right\} \\
& =\exp \left\{\left(\int_{\mathbb{R}_{0}}\left[e^{i c z}-1-i c z\right] \nu(d z)\right) \hat{\Lambda}_{t}^{H}\right\}, \quad c \in \mathbb{R} .
\end{aligned}
$$

Indeed, there is a strong connection between the distributions of $B$ and the Brownian motion, and between $\eta$ and a centered pure jump Lévy process with the same jump behavior. The relationship is based on a random distortion of the time scale. The following characterization is due to [Ser72, Theorem 3.1] (see also [Gri75]).

Theorem 2.3. Let $W_{t}, t \in[0, T]$, be a Brownian motion and $N_{t}, t \in[0, T]$, be a centered pure jump Lévy process with Levy measure $\nu$. Assume that both $W$ and $N$ are independent of $\Lambda$. Then $B$ satisfies A1)-(2.3) and A2) if and only if, for any $t \geq 0$,

$$
B_{t} \stackrel{d}{=} W_{\Lambda_{t}^{B}}
$$

and $\eta$ satisfies A3)(2.4) and A4) if and only if, for any $t \geq 0$,

$$
\eta_{t} \stackrel{d}{=} N_{\hat{\Lambda}_{t}^{H}}
$$

In addition, $B$ is infinitely divisible if $\Lambda^{B}$ is infinitely divisible, and $\eta$ is infinitely divisible if $\hat{\Lambda}^{H}$ is infinitely divisible, see [BNMS06, Theorem 7.1].

\subsection{Stochastic non-anticipating integration and representation theorems}

Let us define $\mathbb{F}^{\mu}=\left\{\mathcal{F}_{t}^{\mu}, t \in[0, T]\right\}$ as the filtration generated by $\mu(\Delta), \Delta \subseteq[0, t] \times \mathbb{R}, t \in[0, T]$. In view of (2.2), A1) and A3), we can see that, for any $t \in[0, T]$,

$$
\mathcal{F}_{t}^{\mu}=\mathcal{F}_{t}^{B} \vee \mathcal{F}_{t}^{H} \vee \mathcal{F}_{t}^{\Lambda},
$$

where $\mathcal{F}_{t}^{B}$ is generated by $B(\Delta \cap[0, T] \times\{0\}), \mathcal{F}_{t}^{H}$ by $H\left(\Delta \cap[0, T] \times \mathbb{R}_{0}\right)$, and $\mathcal{F}_{t}^{\Lambda}$ by $\Lambda(\Delta)$, $\Delta \in[0, t] \times \mathbb{R}$. This is an application of [Win01, Theorem 1] and [DS13, Theorem 2.8]. Set $\mathbb{F}=\left\{\mathcal{F}_{t}, t \in[0, T]\right\}$, where

$$
\mathcal{F}_{t}:=\bigcap_{r>t} \mathcal{F}_{r}^{\mu}
$$

Furthermore, we set $\mathbb{G}=\left\{\mathcal{G}_{t}, t \in[0, T]\right\}$ where $\mathcal{G}_{t}:=\mathcal{F}_{t} \vee \mathcal{F}^{\Lambda}$. Remark that $\mathcal{G}_{T}=\mathcal{F}_{T}, \mathcal{G}_{0}=\mathcal{F}^{\Lambda}$, while $\mathcal{F}_{0}$ is trivial. From now on we set $\mathcal{F}=\mathcal{F}_{T}$.

Lemma 2.4. The filtration $\mathbb{G}$ is right-continuous.

Proof. This can be shown adapting classical arguments for the Lévy case as in e.g. App04, Theorem 2.1.9]. 
For $\Delta \subset(t, T] \times \mathbb{R}$, the conditional independence A2) and A4) means that

$$
\mathbb{E}\left[\mu(\Delta) \mid \mathcal{G}_{t}\right]=\mathbb{E}\left[\mu(\Delta) \mid \mathcal{F}_{t} \vee \mathcal{F}^{\Lambda}\right]=\mathbb{E}\left[\mu(\Delta) \mid \mathcal{F}^{\Lambda}\right]=0
$$

Hence, $\mu$ is a martingale random field (with conditional orthogonal values in $L^{2}(\Omega, \mathcal{F}, \mathbb{P}$ ) with respect to $\mathbb{G}$ in the sense of [DE10] (see Definition 2.1), since

- $\mu$ has a $\sigma$-finite variance measure

$$
m(\Delta):=\mathbb{E}\left[\mu(\Delta)^{2}\right]=\mathbb{E}[\Lambda(\Delta)], \quad \Delta \subseteq \mathbb{X},
$$

with $m(\{0\} \times \mathbb{R})=0$,

- it is additive on pairwise disjoint sets in $\mathcal{B}_{\mathbb{X}}$ and $\sigma$-additive with convergence in $L^{2}$,

- $\mu$ is $\mathbb{G}$-adapted,

- it has the martingale property (2.5),

- $\mu$ has conditionally orthogonal values, if $\Delta_{1}, \Delta_{2} \subset(t, T] \times \mathbb{R}$ such that $\Delta_{1} \cap \Delta_{2}=\emptyset$ then, combining A2), A4), A5) and (2.5),

$$
\mathbb{E}\left[\mu\left(\Delta_{1}\right) \mu\left(\Delta_{2}\right) \mid \mathcal{G}_{t}\right]=\mathbb{E}\left[\mu\left(\Delta_{1}\right) \mid \mathcal{F}^{\Lambda}\right] \mathbb{E}\left[\mu\left(\Delta_{2}\right) \mid \mathcal{F}^{\Lambda}\right]=0
$$

In CW75 there is a discussion about martingale (difference) random fields and the role of ordering associated with the information flow. In their terminology the martingale random fields here treated is both a "strong" and a "weak" martingale.

Denote $\mathcal{I}_{\mathbb{G}}$ as the subspace of $L^{2}\left([0, T] \times \mathbb{R} \times \Omega, \mathcal{B}_{\mathbb{X}} \times \mathcal{F}, \Lambda \times \mathbb{P}\right)$ of the random fields admitting a $\mathbb{G}$-predictable modification, in particular

$$
\|\phi\|_{\mathcal{I}_{\mathbb{G}}}:=\left(\mathbb{E}\left[\int_{0}^{T} \phi_{s}(0)^{2} \lambda_{s}^{B} d s+\int_{0}^{T} \int_{\mathbb{R}_{0}} \phi_{s}(z)^{2} \nu(d z) \lambda_{s}^{H} d s\right]\right)^{\frac{1}{2}}<\infty .
$$

For any $\phi \in \mathcal{I}_{\mathbb{G}}$, we define the (Itô type) non-anticipative stochastic integral $I: \mathcal{I}_{\mathbb{G}} \Rightarrow L^{2}(\Omega, \mathcal{F}, \mathbb{P})$ by

$$
I(\phi):=\int_{0}^{T} \phi_{s}(0) d B_{s}+\int_{0}^{T} \int_{\mathbb{R}_{0}} \phi_{s}(z) \tilde{H}(d s, d z) .
$$

We refer to [DE10] for the details on the integration with respect to martingale random fields of the type discussed here. Recall that $I$ is a linear isometric operator:

$$
\sqrt{E\left[I(\phi)^{2}\right]}=\|I(\phi)\|_{L^{2}(\Omega, \mathcal{F}, \mathbb{P})}=\|\phi\|_{\mathcal{I}_{\mathbb{G}}} .
$$

Because of the structure of the filtration considered, we have the following result (see [DS14]):

Lemma 2.5. Consider $\xi \in L^{2}\left(\Omega, \mathcal{F}^{\Lambda}, \mathbb{P}\right)$ and $\phi \in \mathcal{I}_{\mathbb{G}}$. Then

$$
\xi I(\phi)=I(\xi \phi),
$$

whenever either side of the equality exists as an element in $L^{2}(\Omega, \mathcal{F}, \mathbb{P})$. 
Remark 2.6. It is easy to see that the random field $\mu$ is also a martingale random field with respect to $\mathbb{F}$ and the non-anticipating integration can be done also with respect to $\mathbb{F}$ as for $\mathbb{G}$. $W e$ denote $\mathcal{I}_{\mathbb{F}}$ the corresponding set of integrands. However, results such as Lemma 2.5 and the forthcoming representation would not hold. See also [DS13, Remark 4.4].

We remark that $\left.\mathcal{F}_{t}^{\mu}:=\sigma\{\mu(\Delta), \Delta \subseteq[0, t] \times \mathbb{R}\}=\sigma\left\{I\left(\phi 1_{\Delta}\right), \Delta \subseteq[0, t] \times \mathbb{R}\right), \phi \in \mathcal{I}_{\mathbb{F}}\right\}$ (indeed $\left.\mu(\Delta)=I\left(\mathbf{1}_{\Delta}\right)\right)$ and $\mathcal{G}_{t}:=\sigma\{\mu(\Delta), \Delta \subseteq[0, t] \times \mathbb{R} ; \Lambda(\Delta), \Delta \subseteq[0, T] \times \mathbb{R}\}=\sigma\left\{I\left(\phi 1_{\Delta}\right), \Delta \subseteq\right.$ $\left.[0, t] \times \mathbb{R}), \phi \in \mathcal{I}_{\mathbb{G}}\right\}$

The following representation theorems are given in [DS14].

Theorem 2.7. Integral representation theorem. Assume $\xi \in L^{2}(\Omega, \mathcal{F}, \mathbb{P})$. Then there exists a unique $\phi \in \mathcal{I}_{\mathbb{G}}$ such that

$$
\xi=\mathbb{E}\left[\xi \mid \mathcal{F}^{\Lambda}\right]+\int_{0}^{T} \int_{\mathbb{R}} \phi_{s}(z) \mu(d s, d z) .
$$

Note that the two summands in (2.7) are orthogonal. Here $\mathbb{E}\left[\xi \mid \mathcal{F}^{\Lambda}\right]$ represents the stochastic component of $\xi$ that cannot be recovered by integration on $\mu$.

Remark 2.8. The existence of such a representation is treated in [JS03, Chapter 3], where the result is obtained after a discussion on the solution of the martingale problem. In [DS14], the existence and uniqueness of the above representation is proved by classical density arguments inspired by [Øks05, Section 4] and [Løk05]. In [DS13], the representation is given with respect to $\tilde{H}$ using orthogonal polynomials. There, an explicit formula for the integrand $\phi$ is derived by means of the non-anticipating derivative with respect to $\mathbb{G}$, see [DS13, Theorem 5.1]. This result holds for more general choices of $\Lambda^{H}$, but with an assumption on the moments. The non-anticipating derivative is well-definied with respect to any martingale random-field with orthogonal values and is an operator on the whole $L^{2}(\Omega, \mathcal{F}, \mathbb{P})$. The random variable $\xi^{0}=E\left[\xi \mid \mathcal{F}_{T}^{\Lambda}\right]$ is characterized by having non-anticipating derivative identically null.

There are other related results in the literature, e.g. in [Yab07, Proposition 41] the same representation is proved for a class of Malliavin differentiable random variables (Clark-Ocone type results).

If an $\mathcal{F}_{T}^{H}$-measurable $\xi$ is considered, then an integral representation is given in the general context of (marked) point processes, see for instance [Bré81, Theorem 4.12 and 8.8] or Dav76, BVW75, Jac75]. Theorem 2.7 differs in the choice of filtration, which also leads to different integrals. In [Bré81, Dav76, BBVW75, Jac75], the integrator in the representation theorem is given by $H-\vartheta$, where $\vartheta$ is $\mathbb{F}^{H}$-predictable compensator of $H$. Our $\Lambda^{H}$ is not $\mathbb{F}^{H}$-predictable.

Theorem 2.9. Martingale representation theorem. Assume $M_{t}, t \in[0, T]$, is a $\mathbb{G}$ martingale. Then there exists a unique $\phi \in \mathcal{I}_{\mathbb{G}}$ such that

$$
M_{t}=\mathbb{E}\left[M_{T} \mid \mathcal{F}^{\Lambda}\right]+\int_{0}^{t} \int_{\mathbb{R}} \phi_{s}(z) \mu(d s, d z), \quad t \in[0, T] .
$$

We observe that, in the case we consider $\mu$ to be a martingale random field with respect to $\mathbb{F}$, the corresponding results take a different form. See [DE10]. In particular, we have: 
Theorem 2.10. Integral representation theorem. Assume $\xi \in L^{2}(\Omega, \mathcal{F}, \mathbb{P})$. Then there exists a unique $\phi \in \mathcal{I}_{\mathbb{F}}$ such that

$$
\xi=\xi^{0}+\int_{0}^{T} \int_{\mathbb{R}} \phi_{s}(z) \mu(d s, d z),
$$

where $\xi^{0}$ is a random variable in $L^{2}(\Omega, \mathcal{F}, \mathbb{P})$ orthogonal to the integral part.

In terms of the non-anticipating derivative with respect to $\mathbb{F}$ as studied in [DE10, the random variable $\xi^{0}$ is characterised by having derivative identically null.

\subsection{Backward stochastic differential equations driven by $\mu$}

The problem of hedging considered in this paper leads to different types of BSDEs depending on the information considered. Hereafter, we give an overview of the results needed in the sequel related to both types. In particular, the comparison theorems will play a central role in the solution of the optimisation problem (1.7). Our references are DS14] and [CFS08].

\subsubsection{Information flow $\mathbb{G}$}

In the case of information flow $\mathbb{G}$, the BSDE of reference is of the form:

$$
Y_{t}=\xi+\int_{t}^{T} g_{s}\left(\lambda_{s}, Y_{s}, \phi_{s}\right) d s-\int_{t}^{T} \int_{\mathbb{R}} \phi_{s}(z) \mu(d s, d z), \quad t \in[0, T] .
$$

Given a terminal condition $\xi$ and a driver (or generator) $g$, a solution is given by the couple of $\mathbb{G}$-adapted processes $(Y, \phi)$ on $(\Omega, \mathcal{F}, \mathbb{P})$ satisfying the equation above. Hereafter, we characterise explicitly the functional spaces in use and the elements of the BSDE to obtain a solution. Let $S_{\mathbb{G}}$ be the space of $\mathbb{G}$-adapted stochastic processes $Y_{t}(\omega), t \in[0, T], \omega \in \Omega$, such that

$$
\|Y\|_{S_{\mathbb{G}}}:=\sqrt{\mathbb{E}\left[\sup _{t \in[0, T]}\left|Y_{t}\right|^{2}\right]}<\infty,
$$

and let $\mathcal{H}_{2}^{\mathcal{G}}$ be the space of $\mathbb{G}$-predictable stochastic processes $f_{t}(\omega), t \in[0, T], \omega \in \Omega$, such that

$$
\mathbb{E}\left[\int_{0}^{T} f_{s}^{2} d s\right]<\infty
$$

Denote $\Phi$ the space of functions $\phi: \mathbb{R} \rightarrow \mathbb{R}$ such that

$$
|\phi(0)|^{2}+\int_{\mathbb{R}_{0}} \phi(z)^{2} \nu(d z)<\infty,
$$

where $\nu$ is the jump measure of the market dynamics.

Definition 2.11. We say that $(\xi, g)$ are standard parameters when $\xi \in L^{2}(\Omega, \mathcal{F}, \mathbb{P})$ and $g$ : $[0, T] \times[0, \infty)^{2} \times \mathbb{R} \times \Phi \times \Omega \rightarrow \mathbb{R}$ such that $g$ satisfies the following conditions: 
- $g \cdot(\lambda, Y, \phi, \cdot)$ is $\mathbb{G}$-adapted for all $\lambda \in \mathcal{L}, Y \in S_{\mathbb{G}}, \phi \in \mathcal{I}_{\mathbb{G}}$,

- $g .(\lambda ., 0,0, \cdot) \in \mathcal{H}_{2}^{\mathcal{G}}$, for all $\lambda \in \mathcal{L}$

- there exists $K_{g}>0$ for which

$$
\begin{aligned}
& \left|g_{t}\left(\left(\lambda^{B}, \lambda^{H}\right), y^{(1)}, \phi^{(1)}\right)-g_{t}\left(\left(\lambda^{B}, \lambda^{H}\right), y^{(2)}, \phi^{(2)}\right)\right| \leq K_{g}\left(\left|y^{(1)}-y^{(2)}\right|\right. \\
& \left.+\left|\phi^{(1)}(0)-\phi^{(2)}(0)\right| \sqrt{\lambda^{B}}+\sqrt{\int_{\mathbb{R}_{0}}\left|\phi^{(1)}(z)-\phi^{(2)}(z)\right|^{2} \nu(d z) \sqrt{\lambda^{H}}}\right)
\end{aligned}
$$

- for all $\left(\lambda^{B}, \lambda^{H}\right) \in[0, \infty)^{2}, y^{(1)}, y^{(2)} \in \mathbb{R}$, and $\phi^{(1)}, \phi^{(2)} \in \Phi d t \times d \mathbb{P}$ a.e.

Theorem 2.12. Let $(g, \xi)$ be standard parameters. Then there exists a unique couple $(Y, \phi) \in$ $S_{\mathbb{G}} \times \mathcal{I}_{\mathbb{G}}$ such that

$$
\begin{aligned}
Y_{t} & =\xi+\int_{t}^{T} g_{s}\left(\lambda_{s}, Y_{s}, \phi_{s}\right) d s-\int_{t}^{T} \int_{\mathbb{R}} \phi_{s}(z) \mu(d s, d z) \\
& =\xi+\int_{t}^{T} g_{s}\left(\lambda_{s}, Y_{s}, \phi_{s}\right) d s-\int_{t}^{T} \phi_{s}(0) d B_{s}-\int_{t}^{T} \int_{\mathbb{R}_{0}} \phi_{s}(z) \tilde{H}(d s, d z) .
\end{aligned}
$$

Remark 2.13. The initial point $Y_{0}$ of the solution $Y$ is not necessarily a (deterministic) constant. From the definition of $\mathbb{G}$ and (2.10), we see that $Y_{0}$ is a square integrable $\mathcal{F}^{\Lambda}$-measurable random variable. To be specific, we have:

$$
\begin{aligned}
Y_{0} & =\mathbb{E}\left[\xi+\int_{0}^{T} g_{s}\left(\lambda_{s}, Y_{s}, \phi_{s}\right) d s-\int_{0}^{T} \phi_{s}(0) d B_{s}-\int_{0}^{T} \int_{\mathbb{R}_{0}} \phi_{s}(z) \tilde{H}(d s, d z) \mid \mathcal{F}^{\Lambda}\right] \\
& =\mathbb{E}\left[\xi+\int_{0}^{T} g_{s}\left(\lambda_{s}, Y_{s}, \phi_{s}\right) d s \mid \mathcal{F}^{\Lambda}\right] .
\end{aligned}
$$

For a linear BSDE of the form (2.11), there exists an explicit representation of the solution.

Theorem 2.14. Assume we have the following BSDE:

$$
\begin{aligned}
-d Y_{t}= & {\left[A_{t} Y_{t}+C_{t}+E_{t}(0) \phi_{t}(0) \sqrt{\lambda_{t}^{B}}+\int_{\mathbb{R}_{0}} E_{t}(z) \phi_{t}(z) \nu(d z) \sqrt{\lambda_{t}^{H}}\right] d t } \\
& -\phi_{t}(0) d B_{t}-\int_{\mathbb{R}_{0}} \phi_{t}(z) \tilde{H}(d t, d z), \quad Y_{T}=\xi,
\end{aligned}
$$

where the coefficients satisfy

i) A is a bounded stochastic process, there exists $K_{A}>0$ such that $\left|A_{t}\right| \leq K_{A}$ for all $t \in[0, T]$ $\mathbb{P}$-a.s., 

ii) $C \in \mathcal{H}_{2}^{\mathcal{G}}$,
iii) $E \in \mathcal{I}_{\mathbb{G}}$,
iv) There exists a $K_{E}>0$ such that $0 \leq E_{t}(z)<K_{E} z$ for $z \in \mathbb{R}_{0}$, and $\left|E_{t}(0)\right|<K_{E}$ $d t \times d \mathbb{P}$-a.e.

Then (2.11) has a unique solution $(Y, \phi)$ in $S_{\mathbb{G}} \times \mathcal{I}_{\mathbb{G}}$ and $Y$ has representation

$$
Y_{t}=\mathbb{E}\left[\xi \Gamma_{T}^{t}+\int_{t}^{T} \Gamma_{t}^{s} C_{s} d s \mid \mathcal{G}_{t}\right], \quad t \in[0, T],
$$

where

$$
\begin{aligned}
\Gamma_{s}^{t}:= & \exp \left\{\int_{t}^{s} A_{u}-\frac{1}{2} E_{u}(0)^{2} \mathbf{1}_{\left\{\lambda_{u}^{B} \neq 0\right\}} d u+\int_{t}^{s} E_{u}(0) \frac{\mathbf{1}_{\left\{\lambda_{u}^{B} \neq 0\right\}}}{\sqrt{\lambda_{u}^{B}}} d B_{u}\right. \\
& +\int_{t}^{s} \int_{\mathbb{R}_{0}}\left[\ln \left(1+E_{u}(z) \frac{\mathbf{1}_{\left\{\lambda_{u}^{H} \neq 0\right\}}}{\sqrt{\lambda_{u}^{H}}}\right)-E_{u}(z) \frac{\mathbf{1}_{\left\{\lambda_{u}^{H} \neq 0\right\}}}{\sqrt{\lambda_{u}^{H}}}\right] \nu(d z) \lambda_{u}^{H} d u \\
& \left.+\int_{t}^{s} \int_{\mathbb{R}_{0}} \ln \left(1+E_{u}(z) \frac{\mathbf{1}_{\left\{\lambda_{u}^{H} \neq 0\right\}}}{\sqrt{\lambda_{u}^{H}}}\right) \tilde{H}(d u, d z)\right\}, \quad s \geq t .
\end{aligned}
$$

Note that $\Gamma_{t}^{s}=\frac{\Gamma_{s}^{0}}{\Gamma_{t}^{0}}$.

The next result is a comparison theorem. This result is crucial in the solution of the optimisation problem (1.7).

Theorem 2.15. Comparison theorem. Let $\left(g^{(1)}, \xi^{(1)}\right)$ and $\left(g^{(2)}, \xi^{(2)}\right)$ be two sets of standard parameters for the BSDE's with solutions $\left(Y^{(1)}, \phi^{(1)}\right),\left(Y^{(2)}, \phi^{(2)}\right) \in S_{\mathbb{G}} \times \mathcal{I}_{\mathbb{G}}$. Assume that

$$
g_{t}^{(2)}(\lambda, y, \phi, \omega)=f_{t}\left(y, \phi(0) \kappa(0) \sqrt{\lambda^{B}}, \int_{\mathbb{R}_{0}} \phi(z) \kappa(z) \nu(d z) \sqrt{\lambda^{H}}, \omega\right),
$$

where $\kappa \in \mathcal{I}_{\mathbb{G}}$ satisfies condition iv] from Theorem 2.14 and $f$ is a function $f:[0, T] \times \mathbb{R} \times \mathbb{R} \times$ $\mathbb{R} \times \Omega \rightarrow \mathbb{R}$ which satisfies, for some $K_{f}>0$,

$$
\left|f_{t}(y, b, h)-f_{t}\left(y^{\prime}, b^{\prime}, h^{\prime}\right)\right| \leq K_{f}\left(\left|y-y^{\prime}\right|+\left|b-b^{\prime}\right|+\left|h-h^{\prime}\right|\right),
$$

$d t \times d \mathbb{P}$ a.e. and

$$
\mathbb{E}\left[\int_{0}^{T}\left|f_{t}(0,0,0)\right|^{2} d t\right]<\infty .
$$

If $\xi^{(1)} \leq \xi^{(2)} \mathbb{P}$-a.s. and $g_{s}^{(1)}\left(\lambda_{s}, Y_{s}^{(1)}, \phi_{s}^{(1)}\right) \leq g_{s}^{(2)}\left(\lambda_{s}, Y_{s}^{(1)}, \phi_{s}^{(1)}\right) d t \times d \mathbb{P}$-a.e., then

$$
Y_{t}^{(1)} \leq Y_{t}^{(2)} \quad d t \times d \mathbb{P} \text {-a.e. }
$$




\subsubsection{Information flow $\mathbb{F}$}

In the case of information flow $\mathbb{F}$ the BSDE of reference takes the form:

$$
Y_{t}=\xi+\int_{t}^{T} \int_{\mathbb{R}} f_{s}\left(Y_{s}, \phi_{s}(z)\right)\langle\mu\rangle(d s, d z)-\int_{t}^{T} \int_{\mathbb{R}} \phi_{s}(z) \mu(d s, d z)-N_{T}+N_{t},
$$

where

(i) $\mu(d t, d z), t \in[0, T], z \in \mathbb{R}$, is the $(\mathbb{F}, \mathbb{P})$-martingale random field (2.2),

(ii) $\langle\mu\rangle(d t, d z)$ is its conditional variance measure, see [DE10] Theorem 2.1, which is in fact the correspondent to the conditional quadratic variation for martingales, see [Pro05], and

(iii) $N$, with $N_{0}=0$, is a square integrable $(\mathbb{F}, \mathbb{P})$-martingale orthogonal to $\mu$, i.e. for every set $A \in \mathcal{B}_{\mathbb{R}}$, for $\mu_{t}(A):=\mu((0, t] \times A), t \in[0, T]$, the quadratic variation $[N, \mu(A)]$ is a uniformly integrable martingale.

Moreover, we have

$$
\langle\mu(A)\rangle_{t}=\int_{0}^{t} \int_{A} \lambda_{s}^{B} \delta_{\{0\}}(d z) d s+\int_{0}^{t} \int_{A} 1_{\mathbb{R}_{0}}(z) \nu(d z) \lambda_{s}^{H} d s .
$$

The existence and uniqueness of the solution of (2.12) is treated adapting Proposition 2.1 and Lemma 2.2 of [CFS08] to the martingale random field case. Here we present the variation of these results in the form used later. The definition of standard parameters is analogous to Definition 2.11, but referred to $\mathbb{F}$. The same is intended for the spaces involved. BSDEs of the type (2.12) with standard parameters admit a unique solution, which is characterized by the triple $(Y, \phi, N)$.

Lemma 2.16. Let $a, b, c$ be $\mathbb{F}$-predictable random fields with a bounded and

$$
\mathbb{E}\left[\int_{0}^{T} \int_{\mathbb{R}} b_{s}^{2}(z)\langle\mu\rangle(d s, d z)\right]<\infty .
$$

Let $\mathcal{E}$ be the Doléans exponential of the martingale random field $\int_{0}^{t} \int_{\mathbb{R}} b_{s}(z) \mu(d s, d z), t \in[0, T]$, and define

$$
\psi_{t}:=\exp \left(\int_{0}^{t} \int_{\mathbb{R}} a_{s}(z)\langle\mu\rangle(d s, d z)\right), \quad \Psi_{t}=\psi_{t} \mathcal{E}_{t}, \quad t \in[0, T] .
$$

Suppose that

(i) $\mathcal{E}$ is a positive uniformly integrable martingale;

(ii) $\mathbb{E}\left[\left(\sup _{t \in[0, T]} \psi_{t}\right)^{2} \mathcal{E}_{T}^{2}\right]<\infty$,

(iii) $\mathbb{E}\left[\int_{0}^{T} \int_{\mathbb{R}} \Psi_{s}(z) c_{s}(z)\langle\mu\rangle(d s, d z)\right]<\infty$.

If the linear backward equation

$$
\begin{aligned}
d Y_{t} & =-\int_{\mathbb{R}}\left(a_{t}(z) Y_{t}+b_{t}(z) \phi_{t}(z)+c_{t}(z)\right)\langle\mu\rangle(d s, d z)+\int_{\mathbb{R}} \phi_{t}(z) \mu(d t, d z)+d N_{t} \\
Y_{T} & =\xi
\end{aligned}
$$

has solution $(Y, \phi, N)$ in $S_{\mathbb{F}} \times \mathcal{I}_{\mathbb{F}} \times \mathcal{L}_{\mathbb{F}}^{2, b}$, where $\mathcal{L}_{\mathbb{F}}^{2, b}$ is the space of $L^{2}$-bounded $(\mathbb{F}, \mathbb{P})$-martingales, then $Y$ is given by

$$
Y_{t}=\mathbb{E}\left[\xi \frac{\Psi_{T}}{\Psi_{t}}+\int_{t}^{T} \int_{\mathbb{R}} \frac{\Psi_{s}}{\Psi_{t}} c_{s}(z)\langle\mu\rangle(d s, d z) \mid \mathcal{F}_{t}\right], \quad 0 \leq t \leq T .
$$


Proof. The result presents weaker assumptions on the coefficients compared to Lemma 2.2 in CFS08 which serve better the applications to come. However, the proof follows substantially the same arguments and it will not be detailed here.

Recall that the Doléan exponential is positive if the martingale has jumps grater than -1 . Conditions for uniform integrability can be found in e.g. [LM78.

Remark 2.17. Whenever expression 2.14 makes sense, it is a solution of the linear equation (2.13).

The next result is a comparison theorem for equations of the type (2.12).

Theorem 2.18. Comparison theorem. Consider two linear BSDEs of the form (2.12):

$$
Y_{t}^{(i)}=\xi^{(i)}+\int_{t}^{T} \int_{\mathbb{R}} f_{s}^{(i)}\left(Y_{s}^{(i)}, \phi_{s}^{(i)}\right)\langle\mu\rangle(d s, d z)-\int_{t}^{T} \int_{\mathbb{R}} \phi_{s}^{(i)}(z) \mu(d s, d z)-N_{T}^{(i)}+N_{t}^{(i)},
$$

for $i=1,2$. Define $\delta Y_{t}:=Y_{t}^{(2)}-Y_{t}^{(1)}, \delta \phi_{t}(z):=\phi_{t}^{(2)}(z)-\phi_{t}^{(1)}(z)$, and

$$
\begin{aligned}
a_{t}(z) & :=\frac{f_{t}^{(2)}\left(Y_{t}^{(2)}, \phi_{t}^{(2)}(z)\right)-f_{t}^{(2)}\left(Y_{t}^{(1)}, \phi_{t}^{(2)}(z)\right)}{\delta Y_{t}} 1_{\delta Y_{t} \neq 0}, \\
b_{t}(z) & :=\frac{f_{t}^{(2)}\left(Y_{t}^{(1)}, \phi_{t}^{(2)}(z)\right)-f_{t}^{(2)}\left(Y_{t}^{(1)}, \phi_{t}^{1}(z)\right)}{\delta \phi_{t}(z)} 1_{\delta \phi_{t}(z) \neq 0}, \\
c_{t}(z) & :=f_{t}^{(2)}\left(Y_{t}^{(1)}, \phi_{t}^{(1)}(z)\right)-f_{t}^{(1)}\left(Y_{t}^{(1)}, \phi_{t}^{(1)}(z)\right) .
\end{aligned}
$$

Then the process $\delta Y$ verifies the linear BSDE:

$$
\begin{aligned}
-d \delta Y_{t} & =\int_{\mathbb{R}}\left[a_{t}(z) \delta Y_{t}+b_{t}(z) \delta \phi_{t}(z)+c_{t}(z)\right]\langle\mu\rangle(d t, d z)+\int_{\mathbb{R}} \delta \phi_{t}(z) \mu(d t, d z)-d \delta N_{t}, \\
\delta Y_{T} & =\xi^{(1)}-\xi^{(1)} .
\end{aligned}
$$

Assume that a and $b$ verify condition (i) and (ii) in Lemma 2.16. Assume also that $\xi^{(2)} \geq \xi^{(1)}$ and, for any $t, c_{t}(z) \geq 0 \mathbb{P}-$ a.s. Then, for any $t, Y_{t}^{(2)} \geq Y_{t}^{(1)} \mathbb{P}-$ a.s.

\section{Change of measure: shift transformations}

The various possible scenarios considered are given by all probability measures $\mathbb{Q}$ equivalent to $\mathbb{P}$ obtained by shift transformation, see (1.1). This is chosen as a feasible set of transformations that allow for an explicit evaluation of the Radon-Nikodym density. Hereafter, we study such transformations. We observe that, in the presence of time-change, such transformations do not lead to a self-preserving structure. As illustration we can see that the doubly stochastic Poisson random measure will not be of such structure after the measure change. We also recall that such shift transformations, when applied to Lévy noises, are actually structure preserving.

We introduce the Radon-Nikodym density process $Z_{t}=Z_{t}^{\theta}, t \in[0, T]$, by

$$
\begin{aligned}
& d Z_{t}^{\theta}=Z_{t^{-}}^{\theta}\left(\theta_{t}^{B} d B_{t}+\int_{\mathbb{R}_{0}} \theta_{t}^{H}(z) \tilde{H}(d t, d z)\right), \\
& Z_{0}^{\theta}=1,
\end{aligned}
$$


for $\theta \in \mathcal{I}_{\mathbb{G}}$ with $\theta_{t}(0)=\theta_{t}^{B}$ and $\theta_{t}(z)=\theta_{t}^{H}(z), z \in \mathbb{R}_{0}$, and $\theta_{t}^{H}(z)>-1 \mathbb{P} \times \Lambda$ - a.e. An explicit strong solution of (3.1) is obtained by application of the Itô formula:

$$
\begin{aligned}
Z_{t}^{\theta}= & \exp \left(\int_{0}^{t} \theta_{s}^{B} d B_{s}-\int_{0}^{t} \frac{1}{2}\left(\theta_{s}^{B}\right)^{2} \lambda_{s}^{B} d s\right. \\
& +\int_{0}^{t} \int_{\mathbb{R}_{0}}\left[\ln \left(1+\theta_{s}^{H}(z)\right)-\theta_{s}^{H}(z)\right] \nu(d z) \lambda_{s}^{H} d s \\
& \left.+\int_{0}^{t} \int_{\mathbb{R}_{0}} \ln \left(1+\theta_{s}^{H}(z)\right) \tilde{H}(d s, d z)\right), \quad t \in[0, T] .
\end{aligned}
$$

Since we have assumed $\theta_{t}^{H}(z)>-1 \mathbb{P} \times \Lambda$-a.e., we know that $\ln \left(1+\theta_{s}^{H}(z)\right)$ and the stochastic integration are well-defined. A generalized version of the Novikov condition, see [LM78, ensures uniform integrability of $Z$.

Theorem 3.1. Let $B$ and $\tilde{H}$ be as in Definition 2.1 with respect to $\mathbb{P}$. Assume that $Z_{t}=Z_{t}^{\theta}$, $t \in[0, T]$, with $\theta \in \mathcal{I}_{\mathbb{G}}$, is a positive uniformly integrable $(\mathbb{G}, \mathbb{P})$-martingale with $\mathbb{E}\left[Z_{T}^{2}\right]<\infty$, and define the probability measure $\mathbb{Q}$, equivalent to $\mathbb{P}$, by

$$
\frac{d \mathbb{Q}}{d \mathbb{P}}=Z_{T}
$$

Define $B^{\theta}$ and $\tilde{H}^{\theta}$ by the dynamics

$$
\begin{aligned}
d B_{t}^{\theta} & :=d B_{t}-\theta_{t}^{B} d \Lambda_{t}^{B}, \\
\tilde{H}^{\theta}(d t, d z) & :=\tilde{H}(d t, d z)-\theta_{t}^{H}(z) \Lambda^{H}(d t, d z),
\end{aligned}
$$

where we recall that $\Lambda^{B}(d t,\{0\})=d \Lambda_{t}^{B}=\lambda_{t}^{B} d t$ and $\Lambda^{H}(d t, d z)=\nu(d z) \lambda_{t}^{H} d t$. Moreover, for any bounded predictable $\psi$ such that $\int_{0}^{T} \int_{\mathbb{R}_{0}} \psi_{t}(z) \Lambda^{H}(d t, d z)<\infty, \mathbb{P}-$ a.s., define the process

$$
M_{t}^{\theta}(\psi):=\int_{0}^{t} \int_{\mathbb{R}_{0}} \psi_{s}(z) \tilde{H}^{\theta}(d s, d z), \quad 0 \leq t \leq T
$$

Then $B^{\theta}$ is a continuous $(\mathbb{G}, \mathbb{Q})$-martingale and a time-changed $(\mathbb{G}, \mathbb{Q})$-Brownian motion. Also, $M^{\theta}(\psi)$ is a $(\mathbb{G}, \mathbb{Q})$-martingale, where $\tilde{H}^{\theta}$ is a $(\mathbb{G}, \mathbb{Q})$-martingale random field.

Moreover, if

$$
\mathbb{E}_{\mathbb{Q}}\left[\sup _{t \in[0, T]}\left|\left[B^{\theta}, M^{\theta}(\psi)\right]_{t}\right|\right]<\infty
$$

for $\psi_{t}(z)=1_{\Delta}(t, z), \Delta \in \mathcal{B}_{[0, T] \times \mathbb{R}_{0}}: m(\Delta)=E\left[\Lambda^{H}(\Delta)\right]<\infty$, then $B^{\theta}$ and $M^{\theta}(\psi)$ are strongly orthogonal under $\mathbb{Q}$.

We recall that two $\mathbb{G}$-martingales with values in $L^{2}(\mathbb{Q})$ are strongly orthogonal if their product is a uniformly integrable $(\mathbb{G}, \mathbb{Q})$-martingale or, equivalently, if their quadratic variation process is a uniformly integrable $(\mathbb{G}, \mathbb{Q})$-martingale.

Proof. With $\epsilon \in[0,1]$, define

$$
X_{t}^{\epsilon}:=\epsilon B_{t}^{\theta}+M_{t}^{\theta}(\psi)
$$


We have

$$
d X_{t}^{\epsilon}=-\alpha_{t}^{\epsilon} d t+\epsilon d B_{t}+\int_{\mathbb{R}_{0}} \psi_{t}(z) \tilde{H}(d t, d z)
$$

where

$$
\alpha_{t}^{\epsilon}=\epsilon \theta_{t}^{B} \lambda_{t}^{B}+\int_{\mathbb{R}_{0}} \psi_{t}(z) \theta_{t}^{H}(z) \nu(d z) \lambda_{t}^{H} .
$$

From Lemma 1.27 in ØS07, we know that if $Z_{t} X_{t}^{\epsilon}$ is a local $(\mathbb{G}, \mathbb{P})$-martingale, then $X_{t}^{\epsilon}$ is a local $(\mathbb{G}, \mathbb{Q})$-martingale. From Definition 1.28 and Example 1.29 in [ØS07, and recalling that

$$
d Z_{t}=Z_{t^{-}}\left(\theta_{t}^{B} d B_{t}+\int_{\mathbb{R}_{0}} \theta_{t}^{H}(z) \tilde{H}(d t, d z)\right)
$$

we get the following:

$$
\begin{aligned}
d\left(Z_{t} X_{t}^{\epsilon}\right)= & Z_{t^{-}} d X_{t}^{\epsilon}+X_{t^{-}}^{\epsilon} d Z_{t}+d Z_{t} d X_{t}^{\epsilon} \\
= & Z_{t^{-}}\left(\epsilon+X_{t^{-}}^{\epsilon} \theta_{t}^{B}\right) d B_{t} \\
& +Z_{t^{-}} \int_{\mathbb{R}_{0}}\left(X_{t^{-}}^{\epsilon} \theta_{t}^{H}(z)+\psi_{t}(z)+\psi_{t}(z) \theta_{t}^{H}(z)\right) \tilde{H}(d t, d z) .
\end{aligned}
$$

Thus, $X_{t}^{\epsilon}$ is a local $(\mathbb{G}, \mathbb{Q})$-martingale for all $\epsilon \in[0,1]$. In particular, $X_{t}^{0}=M_{t}^{\theta}(\psi)$ is a local $(\mathbb{G}, \mathbb{Q})$-martingale. Moreover, $B_{t}^{\theta}=X_{t}^{1}-M_{t}^{\theta}(\psi)$ is also a local $(\mathbb{G}, \mathbb{Q})$-martingale.

Since $B^{\theta}$ is a continuous local martingale $\left(B_{0}^{\theta}=0\right)$, with quadratic variation $\left[B^{\theta}, B^{\theta}\right]_{t}=$ $[B, B]_{t}=\Lambda_{t}^{B}$ (the quadratic variation is invariant under equivalent measure change), then $B^{\theta}$ is a time-changed $(\mathbb{G}, \mathbb{Q}$ )-Brownian motion, see Theorem 16.4 in [Kal97]. Hence, it is also a doubly stochastic Gaussian measure as in Definition 2.1.

As for $M^{\theta}(\psi)$, we can see that its quadratic variation is

$$
\left[M^{\theta}(\psi), M^{\theta}(\psi)\right]_{t}=\int_{0}^{t} \int_{\mathbb{R}_{0}} \psi_{s}^{2}(z) H(d s, d z) .
$$

Now, let $\psi_{t}(z)=1_{(0, t] \times B}(t, z)$, for $t \in[0, T]$ and $B \in \mathcal{B}_{\mathbb{R}_{0}}$. Then,

$$
\begin{aligned}
\mathbb{E}_{\mathbb{Q}}\left[\left[M^{\theta}(\psi), M^{\theta}(\psi)\right]_{T}\right] & =\mathbb{E}_{\mathbb{Q}}\left[\int_{0}^{T} \int_{\mathbb{R}_{0}} 1_{(0, t] \times B}(t, z) H(d t, d z)\right] \\
& =\mathbb{E}\left[Z_{T} \int_{0}^{T} \int_{\mathbb{R}_{0}} 1_{(0, t] \times B}(t, z) H(d t, d z)\right] .
\end{aligned}
$$

By Hölder's inequality, we have that

$$
\mathbb{E}_{\mathbb{Q}}\left[\left[M^{\theta}(\psi), M^{\theta}(\psi)\right]_{T}\right] \leq\left(\mathbb{E}\left[Z_{T}^{2}\right]\right)^{1 / 2} \cdot\left(\mathbb{E}\left[H((0, t] \times B)^{2}\right]\right)^{1 / 2}<\infty
$$

Hence $M^{\theta}(\psi)$ is a $(\mathbb{G}, \mathbb{Q})$ - martingale, see e.g. Corollary to Theorem 27.II in Pro05. Denote $\mathcal{B}$ a semi-ring generating $\mathcal{B}_{\mathbb{R}_{0}}$. We can regard the $\sigma$-algebra $\mathcal{B}_{(0, T]}$ as generated by the semi-ring of intervals of the form $(s, t]$, where $0 \leq s<t \leq T$. The $\sigma$-algebra $\mathcal{B}_{(0, T] \times \mathbb{R}_{0}}$ is generated by 
the semi-ring of sets $(s, t] \times A$, where $A \in \mathcal{B}$. For an element $(s, t] \times A$ in the semi-ring, let $\psi=1_{(0, t] \times A}$. Then we have

$$
\tilde{H}^{\theta}((s, t] \times A)=M_{t}^{\theta}(\psi)-M_{s}^{\theta}(\psi) .
$$

By (3.3) and (3.4) $\tilde{H}^{\theta}$ is $\sigma$-finite on the semi-ring $\mathbb{P}$-a.s. (equivalently $\mathbb{Q}$-a.s.), hence we can uniquely extend (3.4) to the $\sigma$-algebra $\mathcal{B}_{[0, T] \times \mathbb{R}_{0}}$, see Theorem 11.3 and Theorem 10.3 in Bil95]. Hence, $\tilde{H}^{\theta}$ has the $(\mathbb{G}, \mathbb{Q})$-martingale property, conditionally orthogonal values with respect to $(\mathbb{G}, \mathbb{Q})$, and its variance measure is $\sigma$-finite. $\tilde{H}^{\theta}$ is clearly $\mathbb{G}$-adapted by its definition, and $\tilde{H}^{\theta}$ is additive and $\sigma$-additive in $L^{2}(\mathbb{Q})$ by its integral form and the condition on $\theta^{H}$. In conclusion, $\tilde{H}^{\theta}$ is a $(\mathbb{G}, \mathbb{Q})$-martingale random field with conditionally orthogonal values.

Finally, we show that $B^{\theta}$ and $M^{\theta}(\psi)$ are strongly orthogonal under $\mathbb{Q}$, for $\psi=1_{\Delta}$ with $\Delta \in$ $\mathcal{B}_{[0, T] \times \mathbb{R}_{0}}: m(\Delta)=\mathbb{E}\left[\Lambda^{H}(\Delta)\right]<\infty$. In fact, observe that

$$
\begin{aligned}
B_{t}^{\theta} & :=B_{t}-\left\langle B, \int_{0} \theta_{s}^{B} d B_{s}\right\rangle_{t} \\
M_{t}^{\theta}(\psi)=\int_{0}^{t} \int_{\mathbb{R}_{0}} 1_{\Delta}(s, z) \tilde{H}^{\theta}(d t, d z):= & \int_{0}^{t} \int_{\mathbb{R}_{0}} 1_{\Delta}(s, z) \tilde{H}(d t, d z) \\
& -\left\langle\int_{0} \int_{\mathbb{R}_{0}} 1_{\Delta}(s, z) \tilde{H}(d s, d z), \int_{0} \int_{\mathbb{R}_{0}} \theta_{s}^{H}(z) \tilde{H}(d s, d z)\right\rangle_{t},
\end{aligned}
$$

Then

$$
\left\langle B^{\theta}, M^{\theta}(\psi)\right\rangle_{t}=\left\langle B, \int_{0}^{\cdot} \int_{\mathbb{R}_{0}} 1_{\Delta}(s, z) \tilde{H}(d t, d z)\right\rangle_{t}=0,
$$

as a consequence of A5) in Definition 2.1. From this we know that $\left[B^{\theta}, M^{\theta}(\psi)\right]_{t}$ is a $\mathbb{Q}$-local martingale and, by (3.2) and Theorem 51.I in Pro05, we get that $\left[B^{\theta}, M^{\theta}(\psi)\right]$ is a uniformly integrable $\mathbb{Q}$-martingale. Then $B_{t}^{\theta}$ and $M_{t}^{\theta}(\psi)$ are strongly orthogonal square integrable martingales.

Remark 3.2. If (3.1) is defined with $\theta \in \mathcal{I}_{\mathbb{F}}$, then $Z^{\theta}$ is an $\mathbb{F}$-adapted process. In this case, the fields $B^{\theta}$ and $\tilde{H}^{\theta}$ would be strongly orthogonal $(\mathbb{F}, \mathbb{Q})$-martingale random fields in the sense discussed above.

Note that $\tilde{H}^{\theta}$ is not a doubly stochastic Poisson random field under $\mathbb{Q}$, in general.

Corollary 3.3. Let $\tilde{H}^{\theta}$ and $Z$ be defined as in Theorem 3.1. If the stochastic field $\theta^{H}$ is deterministic, then $\tilde{H}^{\theta}$ is a $(\mathbb{G}, \mathbb{Q})$-centered doubly stochastic Poisson random field. Moreover, if $\theta_{t}^{H}(z)=\theta_{t}^{H}$, then the new jump measure and the new time distortion process are given by

$$
\nu^{\theta}(d z)=\nu(d z), \quad \lambda_{t}^{\theta}(\omega)=\left\{1+\theta_{t}^{H}\right\} \lambda_{t}(\omega) .
$$

If $\theta_{t}^{H}(z)=\theta^{H}(z)$, then the new jump measure and the new time distortion process are given by

$$
\nu^{\theta}(d z)=\left\{1+\theta^{H}(z)\right\} \nu(d z), \quad \lambda_{t}^{\theta}(\omega)=\lambda_{t}(\omega) .
$$

Proof. This can be shown by studying the characteristic function (under $\mathbb{Q}$ ) of $\tilde{H}^{\theta}(\Delta)$ for $\Delta \in$ $\mathcal{B}_{[0, T] \times \mathbb{R}_{0}}$. 


\section{Hedging under worst case scenario}

In this section we define explicitly the set of scenarios $\mathcal{Q}_{\mathbb{M}}$ considered in the definition of the risk measure in (1.1):

$$
\rho_{t}(\xi):=\operatorname{ess~sup}_{\mathbb{Q} \in \mathcal{Q}_{\mathbb{M}}} \mathbb{E}_{\mathbb{Q}}\left[-\xi \mid \mathcal{M}_{t}\right], \quad t \in[0, T] .
$$

We consider the cases of information flow given by $\mathbb{M}=\mathbb{G}, \mathbb{F}$. Considering the agent's perspective, it is natural to choose the filtration $\mathbb{F}$ as model for the information flow. In fact $\mathbb{G}$ carries the information of the whole process of stochastic time-change, which would be a form of anticipating information embedded in the information flow not reasonably available to an agent. We study the optimisation problem (1.7) in both cases on the market (1.2)-(1.3) with the $\mathbb{F}$ adapted coefficients $r, \alpha, \sigma, \gamma$.

Definition 4.1. Let the process $Z_{t}, t \in[0, T]$, be a $(\mathbb{M}, \mathbb{P})$-martingale defined by

$$
\begin{aligned}
& d Z_{t}=Z_{t^{-}}\left(\theta_{t}^{B} d B_{t}+\int_{\mathbb{R}_{0}} \theta_{t}^{H}(z) \tilde{H}(d t, d z)\right), \\
& Z_{0}=1,
\end{aligned}
$$

for $\theta \in \mathcal{I}_{\mathbb{G}}$ (with the notation $\theta_{t}(0)=\theta_{t}^{B}$ and $\theta_{t}(z)=\theta_{t}^{H}(z)$ for $z \in \mathbb{R}_{0}$ ) and $\theta_{t}^{H}(z)>-1$ $\mathbb{P} \times \Lambda$ - a.e. Consider the cases such that $Z_{T} \in L^{2}(\mathbb{P})$ and (3.2) is satisfied. Moreover, for $K>0$,

$$
\left|\theta_{t}^{B} \lambda_{t}^{B}\right|<K, \quad 0 \leq \theta_{t}^{H}(z) \sqrt{\lambda_{t}^{H}}<K \cdot z, z \in \mathbb{R}_{0}, \quad \mathbb{P} \times d t-\text { a.e. }
$$

Then the set of admissible scenarios is given by:

$$
\mathcal{Q}_{\mathbb{M}}:=\left\{\mathbb{Q} \sim \mathbb{P} \mid \frac{d \mathbb{Q}}{d \mathbb{P}}=Z_{T}^{\theta}, \theta \in \mathcal{I}_{\mathbb{M}}\right\}
$$

where the Radon-Nikodym derivative are of the type above.

We remark that $\mathcal{Q}_{\mathbb{M}}$ is a convex set. Moreover, $\mathcal{Q}_{\mathbb{F}} \subseteq \mathcal{Q}_{\mathbb{G}}$.

We recall that the hedging problem considered (1.6), with the chosen risk measure (1.1), translates to the problem (1.7):

$$
Y_{t}=\underset{\pi \in \Pi_{\mathbb{M}}}{\operatorname{essinf}} \operatorname{esssup}_{\mathbb{Q} \in \mathcal{Q}_{\mathbb{M}}} Y_{t}^{\pi, \mathbb{Q}}
$$

with

$$
\begin{aligned}
Y_{t}^{\pi, \mathbb{Q}}:= & E_{\mathbb{Q}}\left[e^{-\int_{t}^{T} r_{s} d s} F-\int_{t}^{T} e^{-\int_{t}^{s} r_{u} d u} \pi_{s}\left(\alpha_{s}-r_{s}\right) d s\right. \\
& -\int_{t}^{T} e^{-\int_{t}^{s} r_{u} d u} \pi_{s} \sigma_{s} d B_{s} \\
& \left.-\int_{t}^{T} \int_{\mathbb{R}_{0}} e^{-\int_{t}^{s} r_{u} d u} \pi_{s} \gamma_{s}(z) \tilde{H}(d s, d z) \mid \mathcal{M}_{t}\right] .
\end{aligned}
$$

Hence, a solution to (1.7) is given by $(\hat{\pi}, \hat{\mathbb{Q}}) \in \Pi_{\mathbb{M}} \times \mathcal{Q}_{\mathbb{M}}$ such that

$$
\begin{aligned}
Y_{t}=Y_{t}^{\hat{\pi}, \hat{\mathbb{Q}}}=E_{\hat{\mathbb{Q}}}[ & e^{-\int_{t}^{T} r_{s} d s} F-\int_{t}^{T} e^{-\int_{t}^{s} r_{u} d u} \hat{\pi}_{s}\left(\alpha_{s}-r_{s}\right) d s \\
& -\int_{t}^{T} e^{-\int_{t}^{s} r_{u} d u} \hat{\pi}_{s} \sigma_{s} d B_{s} \\
& \left.-\int_{t}^{T} \int_{\mathbb{R}_{0}} e^{-\int_{t}^{s} r_{u} d u} \hat{\pi}_{s} \gamma_{s}(z) \tilde{H}(d s, d z) \mid \mathcal{M}_{t}\right], \quad t \in[0, T] .
\end{aligned}
$$


Analogously, we define the set of admissible portfolio $\Pi_{\mathbb{M}}$ with respect to the filtrations $\mathbb{M}=\mathbb{F}, \mathbb{G}$.

Definition 4.2. The portfolio $\pi:[0, T] \times \Omega \rightarrow \mathbb{R}$ is admissible if

(i) $\pi_{t} \gamma_{t}(z)>-1 \quad \mathbb{P}$-a.s.,

(ii) $\pi$ is $\mathbb{M}$-predictable such that there exists a unique strong càdlàg $\mathbb{M}$-adapted solution $V^{\pi}$ to the dynamics (1.4) on $[0, T]$,

(iii) for all $\mathbb{Q} \in \mathcal{Q}_{\mathbb{M}}$

$$
\mathbb{E}_{\mathbb{Q}}\left[\int_{0}^{T}\left[\left|\alpha_{t}-r_{t}\right|\left|\pi_{t}\right|+\left|\pi_{t} \sigma_{t}\right|^{2} \lambda_{t}^{B}+\int_{\mathbb{R}_{0}}\left|\pi_{t} \gamma_{t}(z)\right|^{2} \nu(d z) \lambda_{t}^{H}\right] d t\right]<\infty .
$$

Note that $\Pi_{\mathbb{M}}$ is a convex set.

The solution to the problem (4.2) is studied via BSDEs and the comparison theorem. The two filtrations lead to different types of BSDEs.

\subsection{Flow of information $\mathbb{G}$}

First of all we consider the filtration $\mathbb{G}$ and the corresponding stochastic process:

$$
\begin{aligned}
& Y_{t}^{\pi, \theta}=\mathbb{E}_{\mathbb{Q}}\left[e^{-\int_{t}^{T} r_{s} d s} F-\int_{t}^{T} e^{-\int_{t}^{s} r_{u} d u} \pi_{s}\left(\alpha_{s}-r_{s}\right) d s\right. \\
& \left.-\int_{t}^{T} e^{-\int_{t}^{s} r_{u} d u} \pi_{s} \sigma_{s} d B_{s}-\int_{t}^{T} \int_{\mathbb{R}_{0}} e^{-\int_{t}^{s} r_{u} d u_{s}} \pi_{s} \gamma_{s}(z) \tilde{H}(d s, d z) \mid \mathcal{G}_{t}\right],
\end{aligned}
$$

where $\pi \in \Pi_{\mathbb{G}}$ and $\mathbb{Q}=\mathbb{Q}^{\theta} \in \mathcal{Q}_{\mathbb{G}}$. By Theorem 3.1, define the $\mathbb{G}$-martingale random fields:

$$
d B_{t}^{\theta}:=d B_{t}-\theta_{t}^{B} \lambda_{t}^{B} d t
$$

and

$$
\tilde{H}^{\theta}(d t, d z):=\tilde{H}(d t, d z)-\theta_{t}^{H}(z) \nu(d z) \lambda_{t}^{H} d t .
$$

So when $\mathbb{M}=\mathbb{G}, Y^{\pi, \mathbb{Q}}$ takes the form:

$$
\begin{aligned}
Y_{t}^{\pi, \theta}= & \mathbb{E}_{\mathbb{Q}}\left[e^{-\int_{t}^{T} r_{s} d s} F-\int_{t}^{T} e^{-\int_{t}^{s} r_{u} d u} \pi_{s}\left(\left(\alpha_{s}-r_{s}\right)+\sigma_{s} \theta_{s}^{B} \lambda_{s}^{B}\right.\right. \\
& \left.+\int_{\mathbb{R}_{0}} \gamma_{s}(z) \theta_{s}^{H}(z) \nu(d z) \lambda_{s}^{H}\right) d s-\int_{t}^{T} e^{-\int_{t}^{s} r_{u} d u} \pi_{s} \sigma_{s} d B_{s}^{\theta} \\
& \left.-\int_{t}^{T} \int_{\mathbb{R}_{0}} e^{-\int_{t}^{s} r_{u} d u} \pi_{s} \gamma_{s}(z) \tilde{H}^{\theta}(d s, d z) \mid \mathcal{G}_{t}\right] \\
= & \mathbb{E}_{\mathbb{Q}}\left[e^{-\int_{t}^{T} r_{s} d s} F-\int_{t}^{T} e^{-\int_{t}^{s} r_{u} d u} \pi_{s}\left(\left(\alpha_{s}-r_{s}\right)+\sigma_{s} \theta_{s}^{B} \lambda_{s}^{B}\right.\right. \\
& \left.\left.+\int_{\mathbb{R}_{0}} \gamma_{s}(z) \theta_{s}^{H}(z) \nu(d z) \lambda_{s}^{H}\right) d s \mid \mathcal{G}_{t}\right] .
\end{aligned}
$$


Hence,

$$
\begin{aligned}
e^{-\int_{0}^{t} r_{u} d u} Y_{t}^{\pi, \theta}=\mathbb{E}_{\mathbb{Q}} & {\left[e^{-\int_{0}^{T} r_{s} d s} F-\int_{0}^{T} e^{-\int_{0}^{s} r_{u} d u} \pi_{s}\right.} \\
& \left.\cdot\left(\left(\alpha_{s}-r_{s}\right)+\sigma_{s} \theta_{s}^{B} \lambda_{s}^{B}+\int_{\mathbb{R}_{0}} \gamma_{s}(z) \theta_{s}^{H}(z) \nu(d z) \lambda_{s}^{H}\right) d s \mid \mathcal{G}_{t}\right] \\
& +\int_{0}^{t} e^{-\int_{0}^{s} r_{u} d u} \pi_{s}\left(\left(\alpha_{s}-r_{s}\right)+\sigma_{s} \theta_{s}^{B} \lambda_{s}^{B}+\int_{\mathbb{R}_{0}} \gamma_{s}(z) \theta_{s}^{H}(z) \nu(d z) \lambda_{s}^{H}\right) d s .
\end{aligned}
$$

The martingale representation Theorem 2.9 applied to

$$
\xi:=e^{-\int_{0}^{T} r_{s} d s} F-\int_{0}^{T} e^{-\int_{0}^{s} r_{u} d u} \pi_{s}\left(\left(\alpha_{s}-r_{s}\right)+\sigma_{s} \theta_{s}^{B} \lambda_{s}^{B}+\int_{\mathbb{R}_{0}} \gamma_{s}(z) \theta_{s}^{H}(z) \nu(d z) \lambda_{s}^{H}\right) d s
$$

gives us the existence of the $\mathbb{G}$-predictable integrands $Z^{\pi, \theta}$ and $U^{\pi, \theta}$ for the two corresponding stochastic integrals, so we have

$$
\begin{aligned}
e^{-\int_{0}^{t} r_{u} d u} Y_{t}^{\pi, \theta} & =\mathbb{E}_{\mathbb{Q}}\left[\xi \mid \mathcal{F}_{T}^{\Lambda}\right]+\int_{0}^{t} Z_{s}^{\pi, \theta} d B_{s}^{\theta}+\int_{0}^{t} \int_{\mathbb{R}_{0}} U_{s}^{\pi, \theta}(z) \tilde{H}^{\theta}(d s, d z) \\
& +\int_{0}^{t} e^{-\int_{0}^{s} r_{u} d u} \pi_{s}\left(\left(\alpha_{s}-r_{s}\right)+\sigma_{s} \theta_{s}^{B} \lambda_{s}^{B}+\int_{\mathbb{R}_{0}} \gamma_{s}(z) \theta_{s}^{H}(z) \nu(d z) \lambda_{s}^{H}\right) d s .
\end{aligned}
$$

The Itô formula allows to obtain the linear BSDE

$$
\begin{aligned}
d Y_{t}^{\pi, \theta}= & \left(r_{t} Y_{t}^{\pi, \theta}+\pi_{t}\left(\alpha_{t}-r_{t}\right)+\left(\pi_{t} \sigma_{t}-e^{\int_{0}^{t} r_{u} d u} Z_{t}^{\pi, \theta}\right) \theta_{t}^{B} \lambda_{t}^{B}\right. \\
& \left.+\int_{\mathbb{R}_{0}}\left(\pi_{t} \gamma_{t}(z)-e^{\int_{0}^{t} r_{u} d u} U_{t}^{\pi, \theta}(z)\right) \theta_{t}^{H}(z) \nu(d z) \lambda_{t}^{H}\right) d t \\
& +e^{\int_{0}^{t} r_{u} d u} Z_{t}^{\pi, \theta} d B_{t}+\int_{\mathbb{R}_{0}} e^{\int_{0}^{t} r_{u} d u} U_{t}^{\pi, \theta}(z) \tilde{H}(d t, d z), \\
Y_{T}^{\pi, \theta}= & F,
\end{aligned}
$$

the solution of which is guaranteed by Theorem 2.14 thanks to (4.1). The generator of this BSDE is:

$$
\begin{aligned}
g \cdot(\lambda, y, z, u(\cdot), \pi, \theta)= & -y r-(\mu-r) \pi-\left(\pi \sigma-e^{\int_{0}^{\cdot} r_{s} d s} z\right) \theta^{B} \lambda^{B} \\
& -\int_{\mathbb{R}_{0}}\left(\pi \gamma \cdot(x)-e^{\int_{0}^{\prime} r_{s} d s} u(x)\right) \theta^{H}(x) \nu(d x) \lambda^{H} .
\end{aligned}
$$

The min-max type of problem corresponding to (4.2) arises in stochastic differential games. With the comparison Theorem 2.15 in hands, we can justify the proof of the following result due to ØS11a in our setting for the driving noises considered in this paper.

As short hand notation, denote $g\left(\pi_{t}, \theta_{t}\right)=g \cdot\left(\lambda, y, z, u(\cdot), \pi_{t}, \theta_{t}\right)$. The solution of a BSDE with standard parameters $(\xi, g)$ is denote by $(Y, Z, U)$, for an optimal $\hat{\theta}$ the solution is denoted by $\left(Y^{\pi}, Z^{\pi}, U^{\pi}\right)$, and for an optimal $\hat{\pi}$ the solution is then $\left(Y^{\theta}, Z^{\theta}, U^{\theta}\right)$. The solution given in the case $g\left(\hat{\pi}_{t}, \hat{\theta}_{t}\right)$ is denoted $(\hat{Y}, \hat{Z}, \hat{U})$.

Theorem 4.3. Let $(\xi, g)$ be standard parameters. Suppose that for all $(\omega, t, \lambda, y, z, u)$ there exist $\hat{\pi}_{t}=\hat{\pi}(\omega, t, \lambda, y, z, u)$ and $\hat{\theta}_{t}=\hat{\theta}(\omega, t, \lambda, y, z, u)$ such that for all admissible portfolios $\pi \in \Pi_{\mathbb{G}}$ and all admissible probability measures $\mathbb{Q}=\mathbb{Q}^{\theta} \in \mathcal{Q}_{\mathbb{G}}$, we have:

$$
g\left(\hat{\pi}_{t}, \theta_{t}\right) \leq g\left(\pi_{t}, \theta_{t}\right) \leq g\left(\pi_{t}, \hat{\theta}_{t}\right),
$$


for a.a. $(\omega, t)$. Assume that the conditions of Theorem 2.15 hold. Suppose $\hat{\pi}$ and $\hat{\theta}$ are admissible, and suppose that for all admissible $(\pi, \theta)$ there exists a unique solution to the BSDE with $\left(\xi, g\left(\pi_{t}, \theta_{t}\right)\right)$ as terminal condition and generator, respectively. Then

$$
\hat{Y}_{t}=Y_{t}^{\hat{\pi}}=\underset{\pi \in \Pi_{\mathbb{G}}}{\operatorname{essinf}} Y_{t}^{\pi}=: Y_{t}=\underset{\mathbb{Q} \in \mathcal{Q}_{\mathbb{G}}}{\operatorname{esssup}}\left\{\operatorname{essinf}_{\pi \in \Pi_{\mathbb{G}}} Y_{t}^{\pi, \theta}\right\}=\underset{\mathbb{Q} \in \mathcal{Q}_{\mathbb{G}}}{\operatorname{ess} \sup _{t}} Y^{\theta}
$$

Proof. The proof is due to ØS11a. By applying the comparison theorem to the solutions of the BSDEs of the couples of standard parameters $\left(F, g\left(\hat{\pi}_{t}, \theta_{t}\right)\right),\left(F, g\left(\pi_{t}, \theta_{t}\right)\right),\left(F, g\left(\pi_{t}, \hat{\theta}_{t}\right)\right)$, by (4.9) we get that $Y_{t}^{\theta} \leq Y_{t}^{\pi, \theta} \leq Y_{t}^{\pi}$, for all admissible $(\pi, \theta)$, thus:

$$
\begin{array}{ll}
\text { For all } \theta: & Y_{t}^{\theta} \leq \underset{\pi \in \Pi}{\operatorname{essinf}} Y_{t}^{\pi, \theta} \quad \mathbb{P} \times d t-\text { a.e. } \\
\text { For all } \pi: & \underset{\theta \in \mathcal{Q}}{\operatorname{esssup}} Y_{t}^{\pi, \theta} \leq Y_{t}^{\pi} \quad \mathbb{P} \times d t-\text { a.e. }
\end{array}
$$

From definition of essential supremum and (4.10), we get

$$
\hat{Y}_{t} \leq \underset{\mathbb{Q} \in \mathcal{Q}_{\mathbb{G}}}{\operatorname{ess} \sup _{t}} Y_{t}^{\theta}=\underset{\mathbb{Q} \in \mathcal{Q}_{\mathbb{G}}}{\operatorname{ess} \sup }\left(\underset{\pi \in \Pi_{\mathbb{G}}}{\operatorname{essinf}} Y_{t}^{\pi, \theta}\right) .
$$

From (4.11) and definition of essential infimum, we get

$$
Y_{t}=\underset{\pi \in \Pi_{\mathbb{G}}}{\operatorname{essinf}}\left\{\underset{\mathbb{Q} \in \mathcal{Q}_{\mathbb{G}}}{\operatorname{ess} \sup _{t}} Y_{t}^{\pi, \theta}\right\} \leq \underset{\pi \in \Pi_{\mathbb{G}}}{\operatorname{essinf}} Y_{t}^{\pi} \leq \hat{Y}_{t}
$$

Hence, we obtain the following chain of inequalities:

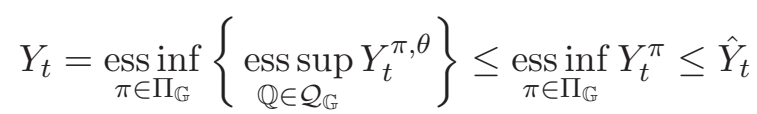

$$
\begin{aligned}
& \leq \operatorname{esssup}_{\mathbb{Q} \in \mathcal{Q}_{\mathbb{G}}} Y_{t}^{\theta} \leq \underset{\mathbb{Q} \in \mathcal{Q}_{\mathbb{G}}}{\operatorname{ess} \sup }\left(\underset{\pi \in \Pi_{\mathbb{G}}}{\operatorname{essinf}} Y_{t}^{\pi, \theta}\right) .
\end{aligned}
$$

Since $\sup (\inf ) \leq \inf (\sup )$ we get equality between all terms.

We shall apply this result. The generator (4.8) satisfies the conditions of $g^{(2)}$ in Theorem 2.15, In fact for an admissible probability measure $\mathbb{Q}^{\theta}$ and an admissible $\pi$, we have:

$$
\begin{aligned}
g_{t}\left(\lambda, y, \zeta, u(\cdot), \pi_{t}, \theta_{t}\right) \\
=-y r_{t}-\pi_{t}\left[\alpha_{t}-r_{t}+\sigma_{t} \theta_{t}^{B} \lambda^{B}+\int_{\mathbb{R}_{0}} \gamma_{t}(z) \theta_{t}^{H}(z) \nu(d z) \lambda^{H}\right] \\
\quad+e^{\int_{0}^{t} r_{s} d s} \zeta \theta_{t}^{B} \sqrt{\lambda^{B}} \sqrt{\lambda^{B}}+\int_{\mathbb{R}_{0}} e^{\int_{0}^{t} r_{s} d s} u(z) \sqrt{\lambda^{H}} \theta_{t}^{H}(z) \nu(d z) \sqrt{\lambda^{H}} .
\end{aligned}
$$

Moreover, condition (4.9) leads to the study of the equations

$$
\frac{\partial g}{\partial \theta^{B}}(\hat{\pi}, \hat{\theta})=0, \quad \frac{\partial g}{\partial \theta^{H}}(\hat{\pi}, \hat{\theta})=0, \quad \frac{\partial g}{\partial \pi}(\hat{\pi}, \hat{\theta})=0 .
$$

The determinant of the Hessian is null, and these equations correspond to a critical point. Recall that $\mathcal{Q}_{\mathbb{G}}$ and $\Pi_{\mathbb{G}}$ are convex. These yield to the characterising equations for the optimal solution.

Summarising, we have the following result. 
Theorem 4.4. Let the reference filtration be $\mathbb{G}$. If $(\hat{\pi}, \hat{\mathbb{Q}}) \in \Pi_{\mathbb{G}} \times \mathcal{Q}_{\mathbb{G}}$ satisfy the equations:

$$
\begin{aligned}
& \left(e^{\int_{0}^{t} r_{s} d s} \hat{Z}_{t}-\hat{\pi}_{t} \sigma_{t}\right) \lambda_{t}^{B}=0, \\
& \int_{\mathbb{R}_{0}}\left(e^{\int_{0}^{t} r_{s} d s} \hat{U}_{t}(z)-\hat{\pi}_{t} \gamma_{t}(z)\right) \nu(d z) \lambda_{t}^{H}=0, \\
& \left(\alpha_{t}-r_{t}\right)+\sigma_{t} \hat{\theta}_{t}^{B} \lambda_{t}^{B}+\int_{\mathbb{R}_{0}} \gamma_{t}(z) \hat{\theta}_{t}^{H}(z) \nu(d z) \lambda_{t}^{H}=0,
\end{aligned}
$$

where $(\hat{Z}, \hat{U}) \in \mathcal{I}_{\mathbb{G}}$ are the integrands in the integral representation (Theorem 2.7):

$$
e^{-\int_{0}^{T} r_{t} d t} F=\mathbb{E}_{\hat{\mathbb{Q}}}\left[e^{-\int_{0}^{T} r_{t} d t} F \mid \mathcal{F}_{T}^{\Lambda}\right]+\int_{0}^{T} \hat{Z}_{s} d B_{s}^{\hat{\theta}}+\int_{0}^{T} \int_{\mathbb{R}_{0}} \hat{U}_{s}(z) \tilde{H}^{\hat{\theta}}(d s, d z)
$$

then $(\hat{\pi}, \hat{\mathbb{Q}})$ is the optimal solution of the problem (1.8). The optimal price process $\hat{Y}_{t}=Y_{t}^{\hat{\pi}, \hat{\mathbb{Q}}}=$ $Y_{t}, t \in[0, T]$, is given by:

$$
\hat{Y}_{t}=\mathbb{E}_{\hat{\mathbb{Q}}}\left[e^{-\int_{t}^{T} r_{s} d s} F-\int_{t}^{T} e^{\int_{0}^{s} r_{u} d u} \hat{Z}_{s} d B_{s}^{\hat{\theta}}-\int_{t}^{T} \int_{\mathbb{R}_{0}} e^{\int_{0}^{s} r_{u} d u} \hat{U}_{s}(z) \tilde{H}^{\hat{\theta}}(d s, d z) \mid \mathcal{G}_{t}\right]
$$

where

$$
d B_{t}^{\hat{\theta}}:=d B_{t}-\hat{\theta}_{t}^{B} \lambda_{t}^{B} d t
$$

is a $(\mathbb{G}, \hat{\mathbb{Q}})$-martingale and a time-changed $(\mathbb{G}, \hat{\mathbb{Q}})$-Brownian motion, and

$$
\tilde{H}^{\hat{\theta}}(d t, d z):=\tilde{H}(d t, d z)-\hat{\theta}_{t}^{H}(z) \nu(d z) \lambda_{t}^{H} d t .
$$

is a $(\mathbb{G}, \hat{\mathbb{Q}})$-martingale random field orthogonal to $B^{\hat{\theta}}$. Under probability measure $\mathbb{P}$, the optimal price process follows the following dynamics:

$$
\begin{aligned}
d \hat{Y}_{t} & =\left(\hat{Y}_{t} r_{t}+\hat{\pi}_{t}\left(\alpha_{t}-r_{t}\right)\right) d t+\hat{\pi}_{t} \sigma_{t} d B_{t}+\int_{\mathbb{R}_{0}} \hat{\pi}_{t} \gamma_{t}(z) \tilde{H}(d t d z) \\
\hat{Y}_{0} & =E_{\hat{\mathbb{Q}}}\left[e^{-\int_{0}^{T} r_{t} d t} F \mid \mathcal{F}_{T}^{\Lambda}\right] .
\end{aligned}
$$

On the other side, if $(\hat{\pi}, \hat{\mathbb{Q}}) \in \Pi_{\mathbb{G}} \times \mathcal{Q}_{\mathbb{G}}$ is an optimal solution, then the equations (4.12)-(4.14) are satisfied.

Remark 4.5. We observe that the optimal strategy $\left(\hat{\pi}, V^{\hat{\pi}}, C^{\hat{\pi}}\right)$ is then given by the process $\hat{\pi}$ as characterised above, the value process $V^{\hat{\pi}}$ from (1.4) has the initial value $v=V_{0}^{\hat{\pi}}=$ $E_{\hat{\mathbb{Q}}}\left[e^{-\int_{0}^{T} r_{t} d t} F\right]$, and the initial cost $C_{0}^{\hat{\pi}}=E_{\hat{\mathbb{Q}}}\left[e^{-\int_{0}^{T} r_{t} d t} F \mid \mathcal{F}_{T}^{\Lambda}\right]-E_{\hat{\mathbb{Q}}}\left[e^{-\int_{0}^{T} r_{t} d t} F\right]$ and is $C_{t}^{\hat{\pi}}=0$ for $t \in(0, T]$.

Remark 4.6. Observe that the optimal $\hat{\mathbb{Q}}$ is a martingale measure for the optimal price process $\hat{Y}$. In fact from (4.16), we have that

$$
e^{-\int_{0}^{t} r_{s} d s} \hat{Y}_{t}=\mathbb{E}_{\hat{\mathbb{Q}}}\left[e^{-\int_{0}^{T} r_{s} d s} \hat{Y}_{T} \mid \mathcal{G}_{t}\right] .
$$




\subsection{Flow of information $\mathbb{F}$}

In the case of flow of information $\mathbb{F}$, we see that the problem (1.7) leads to a different type of BSDE than the one considered so far.

Recall that $\mu(d t, d z)=1_{\{0\}} B(d t, d z)+1_{\mathbb{R}_{0}}(z) \tilde{H}(d t, d z)$, is an $(\mathbb{F}, \mathbb{P})$-martingale random field and that its conditional variance measure is:

$$
\langle\mu\rangle(d t, d z)=\delta_{\{0\}}(d z) \lambda_{s}^{B} d s+1_{\mathbb{R}_{0}}(z) \nu(d z) \lambda_{s}^{H} d s .
$$

Moreover, by Theorem 3.1 we define the $(\mathbb{F}, \mathbb{Q})$ - martingale random field, $\mu^{\theta}$, by

$$
\mu^{\theta}(d t, d z)=\mu(d t, d z)-\beta_{t}(z)\langle\mu\rangle(d t, d z)
$$

where $\beta$ is given by:

$$
\beta_{t}(z):=\theta_{t}^{B} 1_{\{0\}}(z)+\theta_{t}^{H}(z) 1_{\mathbb{R}_{0}}(z)
$$

Note that $\langle\mu\rangle=\left\langle\mu^{\theta}\right\rangle$.

Let us consider the process $Y^{\pi, \mathbb{Q}}$ under filtration $\mathbb{F}$ for $\pi \in \Pi_{\mathbb{F}}$ and $\mathbb{Q} \in \mathcal{Q}_{\mathbb{F}}$ :

$$
\begin{aligned}
Y_{t}^{\pi, \theta}=\mathbb{E}_{\mathbb{Q}}[ & e^{-\int_{t}^{T} r_{s} d s} F-\int_{t}^{T} e^{-\int_{t}^{s} r_{u} d u} \pi_{s}\left(\alpha_{s}-r_{s}\right) d s \\
& -\int_{t}^{T} e^{-\int_{t}^{s} r_{u} d u} \pi_{s} \sigma_{s} d B_{s} \\
& \left.-\int_{t}^{T} \int_{\mathbb{R}_{0}} e^{-\int_{t}^{s} r_{u} d u} \pi_{s} \gamma_{s}(z) \tilde{H}(d s, d z) \mid \mathcal{F}_{t}\right] .
\end{aligned}
$$

Then, by direct computation and using Theorem 2.10, we obtain the following equalities:

$$
\begin{aligned}
e^{-\int_{0}^{t} r_{s} d s} Y_{t}^{\pi, \theta}= & \mathbb{E}_{\mathbb{Q}}\left[e^{-\int_{0}^{T} r_{s} d s} F-\int_{0}^{T} \int_{\mathbb{R}} e^{-\int_{0}^{s} r_{u} d u} \pi_{s}\left[\left(\left(\alpha_{s}-r_{s}\right)+\sigma_{s} \theta_{s}^{B}\right) 1_{\{0\}}(z)\right.\right. \\
& \left.\left.+\gamma_{s}(z) \theta_{s}^{H}(z) 1_{\mathbb{R}_{0}}(z)\right]\left\langle\mu^{\theta}\right\rangle(d s, d z) \mid \mathcal{F}_{t}\right] \\
& +\int_{0}^{t} \int_{\mathbb{R}} e^{-\int_{0}^{s} r_{u} d u} \pi_{s}\left[\left(\left(\alpha_{s}-r_{s}\right)+\sigma_{s} \theta_{s}^{B}\right) 1_{\{0\}}(z)\right. \\
& \left.+\gamma_{s}(z) \theta_{s}^{H}(z) 1_{\mathbb{R}_{0}}(z)\right]\left\langle\mu^{\theta}\right\rangle(d s, d z) \\
= & \mathbb{E}_{\mathbb{Q}}\left[\xi_{0}+\int_{0}^{T} \int_{\mathbb{R}} Z_{t} 1_{\{0\}}(z)+U_{t}(z) 1_{\mathbb{R}_{0}}(z) \mu^{\theta}(d t, d z) \mid \mathcal{F}_{t}\right] \\
& +\int_{0}^{t} \int_{\mathbb{R}} e^{-\int_{0}^{s} r_{u} d u} \pi_{s}\left[\left(\left(\alpha_{s}-r_{s}\right)+\sigma_{s} \theta_{s}^{B}\right) 1_{\{0\}}(z)\right. \\
& \left.+\gamma_{s}(z) \theta_{s}^{H}(z) 1_{\mathbb{R}_{0}}(z)\right]\left\langle\mu^{\theta}\right\rangle(d s, d z) .
\end{aligned}
$$

Equivalently,

$$
\begin{aligned}
e^{-\int_{0}^{t} r_{s} d s} Y_{t}^{\pi, \theta}= & \mathbb{E}_{\mathbb{Q}}\left[\xi_{0} \mid \mathcal{F}_{t}\right]+\int_{0}^{t} \int_{\mathbb{R}} Z_{s} 1_{\{0\}}(z)+U_{s}(z) 1_{\mathbb{R}_{0}}(z) \mu^{\theta}(d s, d z) \\
& +\int_{0}^{t} \int_{\mathbb{R}} e^{-\int_{0}^{s} r_{u} d u} \pi_{s}\left[\left(\left(\alpha_{s}-r_{s}\right)+\sigma_{s} \theta_{s}^{B}\right) 1_{\{0\}}(z)\right. \\
& \left.+\gamma_{s}(z) \theta_{s}^{H}(z) 1_{\mathbb{R}_{0}}(z)\right]\left\langle\mu^{\theta}\right\rangle(d s, d z) .
\end{aligned}
$$


Recall that the random variable $\xi_{0} \in L^{2}(\Omega, \mathcal{F}, \mathbb{Q})$ is orthogonal to the stochastic integrals. The equation above, together with (4.18), yields the BSDE:

$$
\begin{aligned}
d Y_{t}^{\pi, \theta}= & -\int_{\mathbb{R}}\left[\left\{-r_{t} Y_{t}-\pi_{t}\left(\alpha_{t}-r_{t}\right)+\theta_{t}^{B}\left\{e^{\int_{0}^{t} r_{s} d s} Z_{t}-\pi_{t} \sigma_{t}\right\}\right\} 1_{\{0\}}(z)\right. \\
& \left.+\left\{e^{\int_{0}^{t} r_{s} d s} U_{t}(z)-\pi_{t} \gamma_{t}(z)\right\} \theta_{t}^{H}(z) 1_{\mathbb{R}_{0}}(z)\right]\langle\mu\rangle(d t, d z) \\
& +\int_{\mathbb{R}}\left[e^{\int_{0}^{t} r_{s} d s} Z_{t} 1_{\{0\}}(z)+e^{\int_{0}^{t} r_{s} d s} U_{t}(z) 1_{\mathbb{R}_{0}}(z)\right] \mu(d t, d z) \\
& +e^{\int_{0}^{t} r_{s} d s} d \mathbb{E}_{\mathbb{Q}}\left[\xi_{0} \mid \mathcal{F}_{t}\right] \\
Y_{T}^{\pi, \theta}= & F .
\end{aligned}
$$

We remark that $\mathbb{E}_{\mathbb{Q}}\left[\xi_{0} \mid \mathcal{F}_{t}\right], t \in[0, T]$, is an $(\mathbb{F}, \mathbb{Q})$-martingale orthogonal to $\mu^{\theta}$. Thus the process $\int_{0}^{t} e^{\int_{0}^{s} r_{u} d u} d \mathbb{E}_{\mathbb{Q}}\left[\xi_{0} \mid \mathcal{F}_{s}\right], t \in[0, T]$, is an $(\mathbb{F}, \mathbb{Q})$-martingale orthogonal to $\mu^{\theta}$. By direct computation that this process is also an $(\mathbb{F}, \mathbb{P})$-martingale orthogonal to $\mu$. In fact, for $A \in \mathcal{B}(\mathbb{R})$, we have

$$
\begin{aligned}
\left\langle\int_{0} e^{\int_{0}^{s} r_{u} d u} d \mathbb{E}_{\mathbb{Q}}\left[\xi_{0} \mid \mathcal{F}_{s}\right], \mu(A)\right\rangle & =\left\langle\int_{0}^{\cdot} e^{\int_{0}^{s} r_{u} d u} d \mathbb{E}_{\mathbb{Q}}\left[\xi_{0} \mid \mathcal{F}_{s}\right], \mu^{\theta}(A)\right\rangle \\
& +\left\langle\int_{0}^{\cdot} e^{\int_{0}^{s} r_{u} d u} d \mathbb{E}_{\mathbb{Q}}\left[\xi_{0} \mid \mathcal{F}_{s}\right], \int_{0} \int_{\mathbb{R}} \beta_{t}(z) d\langle\mu\rangle(d t, d z)\right\rangle=0 .
\end{aligned}
$$

Another way to look at (4.19) is by application of Theorem 3.1. In fact, setting $\psi_{t}:=e^{-\int_{0}^{t} r_{s} d s}$, $t \in[0, T]$, we have:

$$
\begin{aligned}
\psi_{t} Y_{t}^{\pi, \theta}= & \mathbb{E}_{\mathbb{Q}}\left[\psi_{T} F-\int_{t}^{T} \psi_{s} \pi_{s}\left[\left(\alpha_{s}-r_{s}\right) d s-\sigma_{s} d B_{s}-\int_{\mathbb{R}_{0}} \gamma_{s}(z) \tilde{H}(d s, d z)\right] \mid \mathcal{F}_{t}\right] \\
= & \mathbb{E}_{\mathbb{Q}}\left[\psi_{T} F-\int_{t}^{T} \psi_{s} \pi_{s}\left[\left(\alpha_{s}-r_{s}\right) d s+\sigma_{s}\left(d B_{s}^{\theta}+\theta_{s}^{B} \lambda_{s}^{B} d s\right)\right.\right. \\
& \left.\left.+\int_{\mathbb{R}_{0}} \gamma_{s}(z)\left(\tilde{H}^{\theta}(d s, d z)+\theta_{s}^{H}(z) \nu(d z) \lambda_{s}^{H} d s\right)\right] \mid \mathcal{F}_{t}\right]
\end{aligned}
$$

By use of the martingale property, we get

$$
\begin{aligned}
\psi_{t} Y_{t}^{\pi, \theta}= & \mathbb{E}_{\mathbb{Q}}\left[\psi_{T} F-\int_{t}^{T} \int_{\mathbb{R}} \psi_{s} \pi_{s}\left[\left(\left(\alpha_{s}-r_{s}\right)+\sigma_{s} \theta_{s}^{B}\right) 1_{\{0\}}(z)\right.\right. \\
& \left.\left.+\gamma_{s}(z) \theta_{s}^{H}(z) 1_{\mathbb{R}_{0}}(z)\right]\langle M\rangle(d s, d z) \mid \mathcal{F}_{t}\right] .
\end{aligned}
$$

With $d \mathbb{Q}=Z_{T} d \mathbb{P}$ and $Z_{T}=\mathcal{E}_{T}\left(\int_{0} \int_{\mathbb{R}} \beta_{s}(z) \mu(d s, d z)\right)$, we recognize (4.21) as the solution of the linear BSDE of type, cf. (2.14):

$$
\begin{aligned}
Y_{t}^{\pi, \theta}=\mathbb{E}\left[\frac{\Psi_{T}}{\Psi_{t}} F-\int_{t}^{T} \int_{\mathbb{R}}\right. & \frac{\Psi_{s}}{\Psi_{t}} \pi_{s}\left[\left(\left(\alpha_{s}-r_{s}\right)+\sigma_{s} \theta_{s}^{B}\right) 1_{\{0\}}(z)\right. \\
& \left.\left.+\gamma_{s}(z) \theta^{H}(s, z) 1_{\mathbb{R}_{0}}(z)\right]\langle\mu\rangle(d s, d z) \mid \mathcal{F}_{t}\right] .
\end{aligned}
$$

Here we recall that $r$ is bounded, $\theta \in \mathcal{I}_{\mathbb{F}}$ so that $\mathbb{Q} \in \mathcal{Q}_{\mathbb{F}}$, and (1.5) holds. By Lemma 2.16 and 
Remark 2.17, the $\mathbb{P}$-dynamics correspond to

$$
\begin{aligned}
d Y_{t}^{\pi, \theta}= & -\int_{\mathbb{R}}\left\{-r_{t} 1_{\{0\}}(z) Y_{t}+\theta_{t}^{B} 1_{\{0\}}(z) \bar{Z}_{t}+\theta_{t}^{H}(z) 1_{\mathbb{R}_{0}}(z) \bar{U}_{t}(z)\right. \\
& \left.-\pi_{t}\left[\left(\left(\alpha_{t}-r_{t}\right)+\sigma_{t} \theta_{t}^{B}\right) 1_{\{0\}}(z)+\gamma_{t}(z) \theta_{t}^{H}(z) 1_{\mathbb{R}_{0}}(z)\right]\right\}\langle\mu\rangle(d t, d z) \\
& +\int_{\mathbb{R}} \bar{Z}_{t} 1_{\{0\}}(z)+\bar{U}_{t}(z) 1_{\mathbb{R}_{0}}(z) \mu(d t, d z)+d N_{t} \\
Y_{T}^{\pi, \theta}= & F,
\end{aligned}
$$

where $N$ is an $(\mathbb{F}, \mathbb{P})$-martingale orthogonal to $\mu$. Comparing (4.22) and (4.20). We see that

$$
\begin{aligned}
\bar{Z}_{t} & =e^{\int_{0}^{t} r_{s} d s} Z_{t} \\
\bar{U}_{t}(z) & =e^{\int_{0}^{t} r_{s} d s} U_{t}(z), \\
N_{t} & =\int_{0}^{t} e^{\int_{0}^{s} r_{u} d u} d \mathbb{E}_{\mathbb{Q}}\left[\xi_{0} \mid \mathcal{F}_{s}\right], \quad\left(N_{0}=0\right) .
\end{aligned}
$$

We can state the corresponding result to Theorem 4.3 for the case of information flow $\mathbb{F}$. Set $f\left(\pi_{t}, \theta_{t}\right):=f_{t}\left(\lambda, y, z, u, \pi_{t}, \theta_{t}\right)$ as short-hand notation.

Theorem 4.7. Let $(\xi, f)$ be standard parameters. Suppose that for all $(\omega, t, \lambda, y, z, u)$ there exist $\hat{\pi}_{t}=\hat{\pi}(\omega, t, \lambda, y, z, u)$ and $\hat{\theta}_{t}=\hat{\theta}(\omega, t, \lambda, y, z, u)$ such that, for all admissible portfolios $\pi \in \Pi_{\mathbb{F}}$ and all admissible probability measures $\mathbb{Q}=\mathbb{Q}^{\theta} \in \mathcal{Q}_{\mathbb{F}}$, we have:

$$
f\left(\hat{\pi}_{t}, \theta_{t}\right) \leq f\left(\pi_{t}, \theta_{t}\right) \leq f\left(\pi_{t}, \hat{\theta}_{t}\right)
$$

for a.a. $(\omega, t)$. Assume that the conditions of Theorem 2.18 hold, and that $\hat{\pi}$ and $\hat{\theta}$ are admissible. Suppose that for all admissible $(\pi, \theta)$ there exists a unique solution to the BSDE with $\left(\xi, f\left(\pi_{t}, \theta_{t}\right)\right)$ as terminal condition and generator, respectively. Then

$$
\hat{Y}_{t}=\underset{\pi \in \Pi_{\mathbb{F}}}{\operatorname{essinf}} Y_{t}^{\pi}=: Y_{t}=\underset{\mathbb{Q} \in \mathcal{Q}_{\mathbb{F}}}{\operatorname{ess} \sup }\left\{\underset{\pi \in \Pi_{\mathbb{F}}}{\operatorname{essinf}} Y_{t}^{\pi, \theta}\right\}=\underset{\mathbb{Q} \in \mathcal{Q}_{\mathbb{F}}}{\operatorname{ess} \sup _{t}} Y_{t}^{\theta}
$$

Proof. The argument is the same as for Theorem 4.3, but rely on the comparison theorem in the case $\mathbb{F}$.

From the BSDE in (4.22), we can see that

$$
\begin{aligned}
f_{t}(y, \zeta, u(\cdot), \pi, \theta)=- & \left.-r_{t} y-\pi_{t}\left(\alpha_{t}-r_{t}\right)+\theta^{B}\left(\zeta-\pi_{t} \sigma_{t}\right)\right\} 1_{\{0\}}(z) \\
& +\left\{u(z)-\pi_{t} \gamma_{t}(z)\right\} \theta^{H}(z) 1_{\mathbb{R}_{0}}(z),
\end{aligned}
$$

where $\langle\mu\rangle(d t, d z)=\delta_{\{0\}}(d z) \lambda^{B}(s) d s+1_{\mathbb{R}_{0}}(z) \nu(d z) \lambda^{H}(s) d s$. So we obtain

$$
\begin{aligned}
g .(\lambda, y, \zeta, u(\cdot), \pi, \theta)= & \left.-r y-\pi(\alpha-r)-\theta^{B} \pi \sigma\right\} \lambda^{B}-\int_{\mathbb{R}_{0}} \pi \gamma(z) \theta^{H}(z) \nu(d z) \lambda^{H} \\
& +\theta^{B} \zeta \lambda^{B}+\int_{\mathbb{R}_{0}} \theta^{H}(z) u(z) \nu(d z) \lambda^{H} .
\end{aligned}
$$

We observe that BSDEs of the type (4.22) with (4.23) satisfy the conditions of Theorem 2.18, In the same way as for Theorem 4.4, condition (4.23) yields to the study of saddle points. Hence we obtain the following result: 
Theorem 4.8. Let the reference filtration be $\mathbb{F}$. If $(\hat{\pi}, \hat{\mathbb{Q}}) \in \Pi_{\mathbb{F}} \times \mathcal{Q}_{\mathbb{F}}$ satisfies the equations:

$$
\begin{aligned}
& \left(e^{\int_{0}^{t} r_{s} d s} \hat{Z}_{t}-\hat{\pi}_{t} \sigma_{t}\right) \lambda_{t}^{B}=0, \\
& \int_{\mathbb{R}_{0}}\left(e^{\int_{0}^{t} r_{s} d s} \hat{U}_{t}(z)-\hat{\pi}_{t} \gamma_{t}(z)\right) \nu(d z) \lambda_{t}^{H}=0, \\
& \left(\alpha_{t}-r_{t}\right)+\sigma_{t} \hat{\theta}_{t}^{B} \lambda_{t}^{B}+\int_{\mathbb{R}_{0}} \gamma_{t}(z) \hat{\theta}_{t}^{H}(z) \nu(d z) \lambda_{t}^{H}=0,
\end{aligned}
$$

where $(\hat{Z}, \hat{U}) \in \mathcal{I}_{\mathbb{F}}$ are the integrands in the integral representation:

$$
e^{-\int_{0}^{T} r_{t} d t} F=\hat{\xi}_{0}+\int_{0}^{T} \hat{Z}_{s} d B_{s}^{\hat{\theta}}+\int_{0}^{T} \int_{\mathbb{R}_{0}} \hat{U}_{s}(z) \tilde{H}^{\hat{\theta}}(d s, d z)
$$

and $\hat{\xi}_{0} \in L^{2}(\Omega, \mathcal{F}, \hat{\mathbb{Q}})$ is a random variable orthogonal to the stochastic integrals (cf. Theorem 2.10), then $(\hat{\pi}, \hat{\mathbb{Q}})$ is the solution of the optimisation problem (4.2). The optimal price process $\hat{Y}_{t}=Y_{t}^{\hat{\pi}, \hat{\mathbb{Q}}}=Y_{t}, t \in[0, T]$, is given by:

$$
\hat{Y}_{t}=\mathbb{E}_{\hat{\mathbb{Q}}}\left[e^{-\int_{t}^{T} r_{s} d s} F-\int_{t}^{T} e^{\int_{0}^{s} r_{u} d u} \hat{Z}_{s} d B_{s}^{\hat{\theta}}-\int_{t}^{T} \int_{\mathbb{R}_{0}} e^{\int_{0}^{s} r_{u} d u} \hat{U}_{s}(z) \tilde{H}^{\hat{\theta}}(d s, d z) \mid \mathcal{F}_{t}\right],
$$

where

$$
d B_{t}^{\hat{\theta}}:=d B_{t}-\hat{\theta}_{t}^{B} \lambda_{t}^{B} d t
$$

and

$$
\tilde{H}^{\hat{\theta}}(d t, d z):=\tilde{H}(d t, d z)-\hat{\theta}_{t}^{H}(z) \nu(d z) \lambda_{t}^{H} d t .
$$

These are orthogonal $(\mathbb{F}, \hat{\mathbb{Q}})$-martingale random fields. Under probability measure $\mathbb{P}$, the optimal price process has the following dynamics:

$$
\begin{aligned}
d \hat{Y}_{t} & =\left(\hat{Y}_{t} r_{t}+\hat{\pi}_{t}\left(\alpha_{t}-r_{t}\right)\right) d t+\hat{\pi}_{t} \sigma_{t} d B_{t}+\int_{\mathbb{R}_{0}} \hat{\pi}_{t} \gamma_{t}(z) \tilde{H}(d t, d z)+e^{\int_{0}^{t} r_{s} d s} d \mathbb{E}_{\hat{\mathbb{Q}}}\left[\hat{\xi}_{0} \mid \mathcal{F}_{t}\right] \\
\hat{Y}_{0} & =E_{\hat{\mathbb{Q}}}\left[e^{-\int_{0}^{T} r_{t} d t} F\right] .
\end{aligned}
$$

Remark 4.9. We observe that the optimal strategy $\left(\hat{\pi}, V^{\hat{\pi}}, C^{\hat{\pi}}\right)$ is then given by the process $\hat{\pi}$ as characterised above. The wealth $V^{\hat{\pi}}$ on the market has the initial value $V_{0}^{\hat{\pi}}=v=E_{\hat{\mathbb{Q}}}\left[e^{-\int_{0}^{T} r_{t} d t} F\right]$, and the cost is a process $C_{t}^{\hat{\pi}}, t \in[0, T]$, given as follows. Observe that

$$
d \hat{Y}_{t}=d V_{t}^{\hat{\pi}}+\left(\hat{Y}_{t}-V_{t}^{\hat{\pi}}\right) r_{t} d t+e^{\int_{0}^{t} r_{s} d s} d \mathbb{E}_{\hat{\mathbb{Q}}}\left[\hat{\xi}_{0} \mid \mathcal{F}_{t}\right]
$$

then

$$
d C_{t}^{\hat{\pi}}=C_{t}^{\hat{\pi}} r_{t} d t+e^{\int_{0}^{t} r_{s} d s} d \mathbb{E}_{\hat{\mathbb{Q}}}\left[\hat{\xi}_{0} \mid \mathcal{F}_{t}\right] ; \quad C_{0}^{\hat{\pi}}=0 .
$$

which results in $C_{t}^{\hat{\pi}}=e^{\int_{0}^{t} r_{s} d s}\left(\mathbb{E}_{\hat{\mathbb{Q}}}\left[\hat{\xi}_{0} \mid \mathcal{F}_{t}\right]-\mathbb{E}_{\hat{\mathbb{Q}}}\left[\hat{\xi}_{0}\right]\right)$.

Remark 4.10. Observe that the optimal $\hat{\mathbb{Q}}$ is a martingale measure for the optimal price process $\hat{Y}$. In fact, the price process is given by:

$$
e^{-\int_{0}^{t} r_{s} d s} \hat{Y}_{t}=\mathbb{E}_{\hat{\mathbb{Q}}}\left[e^{-\int_{0}^{T} r_{s} d s} \hat{Y}_{T} \mid \mathcal{F}_{t}\right] .
$$

Remark 4.11. The characterizing equations (4.12)-(4.14) and (4.25)-(4.27) are formally the same, being the difference on the measurability properties of the processes involved. Denote $\hat{\mathbb{Q}}_{\mathbb{G}}=\mathbb{Q}^{\hat{\pi}} \in \mathcal{Q}_{\mathbb{G}}$ in Theorem 4.4 and $\hat{\mathbb{Q}}_{\mathbb{F}}=\mathbb{Q}^{\hat{\pi}} \in \mathcal{Q}_{\mathbb{F}}$ in Theorem 4.8. From equations (4.14) and (4.27) combined with Theorem [3.1, we can see that $\hat{\mathbb{Q}}_{\mathbb{F} \mid \mathbb{F}}=\hat{\mathbb{Q}}_{\mathbb{F}}$. 


\section{Conclusions and example}

With the intent of finding a hedging strategy in the incomplete market (1.2)-(1.3) we have studied the optimization problem (1.6). We have developed the solution using BSDEs and their comparison theorems. for this we refer to DS14] and [CFS08], of which we adapt the results. For our approach it is crucial the result of [ØS11a] developed for stochastic differential games. Indeed we have transformed the hedging problem under model uncertainty in a min-max type problem (1.7) by exploiting the explicit representation of the risk-measure considered (1.1).

The noises considered are naturally linked to two different filtrations. The filtration $\mathbb{G}$ captures all the statistical properties of the noises, allowing to exploit the underlying Gaussian and Poisson structure, see Definition 2.1. The filtration $\mathbb{F}$ is substantially the filtration generated by the noises. We study the problem (1.7) with respect to both situations and we observe that $\mathcal{F}_{T}=\mathcal{G}_{T}$. Correspondingly, we have proposed two BSDEs related to the the two filtered probability spaces. The terminal condition is the same. The results obtained show differences in terms of adaptability of the solutions and the structure of the solution itself, cf. (4.17) and (4.30).

Both set-ups lead to hedging strategies $\left(\hat{\pi}, V^{\hat{\pi}}, C^{\hat{\pi}}\right)$ with presence of cost process. In the case of $\mathbb{G}$, the cost process accounts for the anticipated knowledge of the time-change. In the case of $\mathbb{F}$, the cost process represents the spread between the perfect hedge and the best self-financing strategy. Here the distances are evaluated in terms of the risk-measure (1.1).

From the methodological point of view, we remark that the BSDEs presented for the study in the case of filtration $\mathbb{F}$ are based on the properties of the martingale random fields and we recall that our noises are martingale random fields with respect to both filtrations. In fact in this framework of general martingales we can see the correspondence between the two set-ups generated by the two filtrations.

As explained earlier, from a financial modeling perspective it is better suited to consider the information flow given by $\mathbb{F}$.

Example 5.1. Toy example. Let $e^{-\int_{0}^{T} r_{t} d t} F$ is $\mathcal{F}_{T}^{\Lambda}$-measurable ( $r$ deterministic). In the information flow $\mathbb{G}$, the integral representation (4.15) shows that the optimal integrands $(\hat{Z}, \hat{U}) \in \mathcal{I}_{\mathbb{G}}$ are null and that the optimal strategy presents $\hat{\pi}=0$, and $v=\mathbb{E}_{\hat{\mathbb{Q}}_{\mathbb{G}}}\left[e^{-\int_{0}^{T} r_{t} d t} F\right]$. Here the values $\left(\hat{\theta}^{B}, \hat{\theta}^{H}\right)$ for the probability measure $\hat{\mathbb{Q}}_{\mathbb{G}}=\mathbb{Q}^{\hat{\theta}} \in \mathcal{Q}_{\mathbb{G}}$ are given by (4.14). See Theorem 4.4. The cost process is $C_{0}^{\hat{\pi}}=e^{-\int_{0}^{T} r_{t} d t} F-\mathbb{E}_{\hat{\mathbb{Q}}_{\mathbb{G}}}\left[e^{-\int_{0}^{T} r_{t} d t} F\right], C_{t}^{\hat{\pi}}=0, t \in(0, T]$.

Consider the case of information flow $\mathbb{F}$. Denote $\mathbb{H}_{\mathbb{F}} \subset L^{2}\left(\Omega, \mathcal{F}, \hat{\mathbb{Q}}_{\mathbb{F}}\right)$ and $\mathbb{H}_{\mathbb{G}} \subset L^{2}\left(\Omega, \mathcal{F}, \hat{\mathbb{Q}}_{\mathbb{G}}\right)$ the spaces generated by the integrals $\int_{0}^{T} \int_{\mathbb{R}} \phi_{t}(z) \mu(d t, d z)$ for all $\phi \in \mathcal{I}_{\mathbb{F}}$ and $\phi \in \mathcal{I}_{\mathbb{G}}$, respectively. Being $\hat{\mathbb{Q}}_{\mathbb{F}}=\hat{\mathbb{Q}}_{\mathbb{G} \mid \mathbb{F}}$ (see Remark 4.11], then $\mathbb{H}_{\mathbb{F}} \subset \mathbb{H}_{\mathbb{G}}$. Hence,

$$
L^{2}\left(\Omega, \mathcal{F}, \hat{\mathbb{Q}}_{\mathbb{G}}\right) \ominus \mathbb{H}_{\mathbb{F}} \supset L^{2}\left(\Omega, \mathcal{F}, \hat{\mathbb{Q}}_{\mathbb{G}}\right) \ominus \mathbb{H}_{\mathbb{G}} \ni e^{-\int_{0}^{T} r_{t} d t} F .
$$

Hence $\hat{\xi}_{0}=e^{-\int_{0}^{T} r_{t} d t} F$ and $(\hat{Z}, \hat{U}) \equiv 0$ in the representation (4.28). The optimal strategy is then $\hat{\pi}=0, v=\mathbb{E}_{\hat{\mathbb{Q}}_{\mathbb{F}}}\left[e^{-\int_{0}^{T} r_{t} d t} F\right]$, and $C_{t}^{\hat{\pi}}=\mathbb{E}_{\hat{\mathbb{Q}}_{\mathbb{F}}}\left[e^{-\int_{0}^{T} r_{t} d t} F \mid \mathcal{F}_{t}\right]-\mathbb{E}_{\hat{\mathbb{Q}}_{\mathbb{F}}}\left[e^{-\int_{0}^{T} r_{t} d t} F\right], t \in[0, T]$.

In line with Remarks 4.6 and 4.10 , we see that the optimal measures $\mathbb{Q}_{\mathbb{F}}$ and $\mathbb{Q}_{\mathbb{G}}$ are risk-neutral in the given market. In fact, applying (4.14) or (4.27), i.e.

$$
\left(\alpha_{t}-r_{t}\right)+\sigma_{t} \hat{\theta}_{t}^{B}+\int_{\mathbb{R}_{0}} \gamma_{t}(z) \hat{\theta}_{t}^{H}(z) \nu(d z) \lambda_{t}=0
$$


we have

$$
\begin{aligned}
d\left(e^{-\int_{0}^{t} r_{s} d s} S_{t}^{(1)}\right) & =e^{-\int_{0}^{t} r_{s} d s} S_{t}^{(1)}\left[\left(\alpha_{t}-r_{t}\right) d t+\sigma_{t} d B_{t}+\int_{\mathbb{R}_{0}} \gamma_{t}(z) \tilde{H}(d t, d z)\right] \\
& =e^{-\int_{0}^{t} r_{s} d s} S_{t}^{(1)}\left[\sigma_{t} d B_{t}^{\hat{\theta}}+\int_{\mathbb{R}_{0}} \gamma_{t}(z) \tilde{H}^{\hat{\theta}}(d t, d z)\right] .
\end{aligned}
$$

This result is consistent with the observations of ØS11b in the context of dynamics given by a jump diffusion and in the literature related to risk-minimizing strategies.

\section{Acknowlegdements}

We acknowledge the support of the Centre of Advanced Study (CAS) at the Norwegian Academy of Science and Letters that has hosted and funded the research project Stochastics in Environmental and Financial Economics (SEFE) during the academic year 2014/15.

\section{References}

[App04] David Applebaum. Lévy Processes and Stochastic Calculus. Cambrigde University press, 2004.

[Bil95] Patrick Billingsley. Probability and measure. Wiley Series in Probability and Mathematical Statistics. John Wiley \& Sons, Inc., New York, third edition, 1995. A Wiley-Interscience Publication.

[BNMS06] Ole Barndorff-Nielsen, Makoto Maejima, and Ken-iti Sato. Infinite divisibility for stochastic processes and time change. Journal of Theoretical Probability, 19(2):411446, 2006.

[BNNS02] Ole E. Barndorff-Nielsen, Elisa Nicolato, and Neil Shephard. Some recent developments in stochastic volatility modelling. Quantitative Finance, 2(1):11-23, 2002.

[BP15] Anne Balter and Antoon Pelsser. Pricing and hedging in incomplete markets with model ambiguity. SSRN 2459405, January 2015.

[Bré81] Pierre Brémaud. Point Processes and Queues - Martingale dynamics. Springer, 1981.

[BVW75] René Boel, Pravin Varaiya, and Eugene Wong. Martingales on jump processes I. Representation results. SIAM Journal on control, 13(5), 1975.

[CCR14] Claudia Ceci, Alessandra Cretarola, and Francesco Russo. BSDEs under partial information and financial applications. Stochastic Process. Appl., 124(8):2628-2653, 2014.

[CE02] Zengjing Chen and Larry Epstein. Ambiguity, risk, and asset returns in continuous time. Econometrica, 70(4):1403-1443, 2002.

[CFS08] Raffaella Carbone, Benedetta Ferrario, and Marina Santacroce. Backward stochastic differential equations driven by càdlàg martingales. Theory of Probability and its Applications, 52(2):304-314, 2008.

[CGMY03] Peter Carr, Heliète German, Dilip Madan, and Marc Yor. Stochastic volatility for Lévy processes. Mathematical Finance, 13(3):345-282, 2003. 
[CW75] R. Cairoli and John B. Walsh. Stochastic integrals in the plane. Acta Math., 134:111$183,1975$.

[Dav76] Mark H. A. Davis. The representation of martingales of jump processes. SIAM Journal on control and optimization, 14(4):623-638, 1976.

[DE10] Giulia Di Nunno and Inga B. Eide. Minimal-variance hedging in large financial markets: random fields approach. Stochastic Analysis and Applications, 28(1):5485,2010 .

[Del12] Lukasz Delong. No-good-deal, local mean variance, ambiguity pricing and hedging of an insurance payment process. ASTIN Bulletin, 42:203-232, 2012.

[Del13] Lukasz Delong. Backward Stochastic Differential Equations with Jumps and their Actuarial and Financial Applications. European Actuarial Academy Series. Springer-Verlag, 2013.

[DR07] Giulia Di Nunno and Yuri Rozanov. Stochastic integrals and adjoint derivatives. In F.E. Benth, G. Di Nunno, T. Lindstrøm, B. Øksendal, and T. Zhang, editors, Stochastic Analysis and Applications, volume 2 of Abel Symposia, pages 265-307. Springer Berlin Heidelberg, 2007.

[DS13] Giulia Di Nunno and Steffen Sjursen. On chaos representation and orthogonal polynomials for the doubly stochastic Poisson process. In Robert C. Dalang, Marco Dozzi, and Francesco Russo, editors, Seminar on Stochastic Analysis, Random Fields and Applications VII, volume 67 of Progress in Probability, pages 23-54. Springer Basel, 2013.

[DS14] Giulia Di Nunno and Steffen Sjursen. BSDEs driven by time-changed Lévy noises and optimal control. Stochastic Processes and their Applications, 124:1679-1709, 2014.

[Gri75] Bronius Grigelionis. Characterization of stochastic processes with conditionally independent increments. Lithuanian Mathematical Journal, 15(4):562-567, 1975.

[Hes93] Steven L Heston. A closed-form solution for options with stochastic volatility with applications to bond and currency options. Review of financial studies, 6(2):327-343, 1993.

[HW87] John Hull and Alan White. The pricing of options on assets with stochastic volatilities. The journal of finance, 42(2):281-300, 1987.

[Jac75] Jean Jacod. Multivariate point processes: Predictable projection, Radon-Nikodym derivatives, representation of martingales. Probability Theory and Related Fields, 31(3):235-253, 1975.

[Jac79] Jean Jacod. Existence and uniqueness for stochastic differential equations. In M. Kohlmann and W. Vogel, editors, Stochastic Control Theory and Stochastic Differential Systems, volume 16 of Lecture Notes in Control and Information Sciences, pages 435-446. Springer Berlin / Heidelberg, 1979.

[JMSS12] Monique Jeanblanc, Michael Mania, Marina Santacroce, and Martin Schweizer. Mean-variance hedging via stochastic control and BSDEs for general semimartingales. The Annals of Applied Probability, 22(6):2388-2428, 2012. 
[JS03] Jean Jacod and Albert N. Shiryaev. Limit Theorems for Stochastic Processes. Springer, 2003.

[Kal97] Olav Kallenberg. Foundations of Modern Probability. Springer, 1997.

[Kar14] Erik H. Karlsen. Optimal portfolio problems under model ambiguity. Thesis, University of Oslo, May 2014.

[Lan98] David Lando. On Cox processes and credit risky securities. Review of Derivatives Research, 2(2-3):99-120, 1998.

[Lim05] Andrew Lim. Mean-variance hedging when there are jumps. Siam J. Control Optim., 44(5):1893-1922, 2005.

[LM78] Dominique Lépingle and Jean Mémin. Sur l'intégrabilité uniforme des martingales exponentielles. Z. Wahrsch. Verw. Gebiete, 42(3):175-203, 1978.

[Løk05] Arne Løkka. Martingale Representation of Lévy Processes. Stochastic Analysis and Applications, 22(4):867-892, 2005.

[Øks05] Bernt Øksendal. Stochastic Differential Equations. Springer, 2005.

[ØS07] Bernt Øksendal and Agnès Sulem. Applied stochastic control of jump diffusions. Universitext. Springer, Berlin, second edition, 2007.

[ØS11a] Bernt Øksendal and Agnès Sulem. Portfolio optimization under model uncertainty and BSDE games. Quant. Finance, 11(11):1665-1674, 2011.

[ØS11b] Bernt Øksendal and Agnès Sulem. Robust stochastic control and equivalent martingale measures. In Stochastic analysis with financial applications, volume 65 of Progr. Probab., pages 179-189. Birkhäuser/Springer Basel AG, Basel, 2011.

[Pro05] Philip Protter. Stochastic Integration and Differential Equations. Springer, 2005. Version 2.1.

[Ser72] Richard F. Serfozo. Processes with conditional stationary independent increments. Journal of applied probability, 9(2), 1972.

[SS91] Elias M Stein and Jeremy C Stein. Stock price distributions with stochastic volatility: an analytic approach. Review of financial Studies, 4(4):727-752, 1991.

[Win01] Matthias Winkel. The recovery problem for time-changed Lévy processes. Research Report MaPhySto 2001-37, October 2001.

[Yab07] Aleh L. Yablonski. The Malliavin calculus for processes with conditionally independent increments. In F.E. Benth, G. Nunno, T. Lindstrøm, B. Øksendal, and T. Zhang, editors, Stochastic Analysis and Applications, volume 2 of Abel Symposia, pages 641-678. Springer Berlin Heidelberg, 2007. 